Collection SFN 11 (2010) 101-126

(C) Owned by the authors, published by EDP Sciences, 2010

DOI: $10.1051 / \mathrm{sfn} / 201011007$

\title{
Dynamique et matière molle
}

\author{
R. Zorn
}

IFF, Forschungszentrum Jülich, 52425 Jülich, Allemagne

\begin{abstract}
Résumé. La diffusion inélastique de neutrons est un outil puissant pour observer la dynamique en matière molle. Elle fournit une information combinée sur la structure et la dynamique des processus microscopiques. En raison de la longueur d'onde et de l'énergie des neutrons utilisés, il est possible d'accéder aux échelles de temps et de longueur pertinentes dans l'étude des systèmes de la matière molle. Dans la première partie de cet article trois techniques seront décrites qui sont particulièrement utiles pour la recherche en matière molle: la spectroscopie par temps-de-vol, la spectroscopie de rétrodiffusion et la spectroscopie par écho de spin. Dans la seconde partie, une application particulière (la dynamique des polymères) sera discutée en détail.
\end{abstract}

\section{DIFFUSION INÉLASTIQUE DE NEUTRONS}

Afin d'étudier les processus dynamiques en matière molle qui s'étalent sur de larges échelles de temps, il est nécessaire de combiner différentes techniques de diffusion inélastique de neutrons (INS). La figure 1 montre les échelles de temps et les résolutions spatiales des différentes méthodes d'INS et les compare avec les échelles accessibles par d'autres techniques expérimentales, de diffusion (lumière, rayons X) ou non (RMN).

La diffusion inélastique de neutrons est un outil indispensable à l'observation de processus ayant lieu à l'échelle moléculaire (1 ...10 ̊) sur des échelles de temps microscopiques (ps...ns). La diffusion de lumière permet d'accéder à une gamme de temps plus large, mais du fait de la longueur d'onde de la lumière, sa résolution spatiale est limitée à des objets beaucoup plus grands que les molécules (e.g. les fluctuations de concentration de particules colloïdales). Des développements récents de diffusion inélastique des rayons $\mathrm{X}$ sur les sources synchrotron intenses permettent l'observation de processus très rapides $(<\mathrm{ps})$ par spectroscopie conventionnelle et de processus lents par corrélation de photons.

L'objectif d'une expérience d'INS est, comme pour les autres techniques de diffusion inélastique, de mesurer les transferts de quantité de mouvement $\mathbf{q}=\mathbf{k}_{\mathrm{f}}-\mathbf{k}_{\mathrm{i}}$ et d'énergie $\hbar \omega=E_{\mathrm{f}}-E_{\mathrm{i}}$. Ici, $\mathbf{k}_{\mathrm{f}}$ et $\mathbf{k}_{\mathrm{i}}$ sont respectivement les vecteurs d'onde $(k=2 \pi / \lambda)$ et $E_{\mathrm{f}}, E_{\mathrm{i}}$ les énergies des neutrons diffusés (final) et incidents (initial).

Ceci requiert généralement un monochromateur pour le faisceau de neutrons incident et un analyseur pour les neutrons diffusés. Dans le montage le plus simple, le spectromètre 3-axes, on utilise les plans de Bragg de cristaux de la même manière que des plans de diffraction en spectroscopie optique (figure 2).

Bien que les spectromètres 3-axes soient encore utilisés dans des études pour lesquelles une très bonne résolution en $q$ est nécessaire (essentiellement des cristaux présentant des pics de Bragg), d'autres types d'instruments se sont révélés plus performants pour l'étude des systèmes de la "matière molle" (e.g. les liquides et les verres). Les plus importants vont être présentés ici : le spectromètre à Temps-deVol (TDV), le spectromètre de rétrodiffusion, ou backscattering (BS) et le spectromètre à écho de spin de neutrons (NSE).

This is an Open Access article distributed under the terms of the Creative Commons Attribution-Noncommercial License 3.0, which permits unrestricted use, distribution, and reproduction in any noncommercial medium, provided the original work is properly cited. 


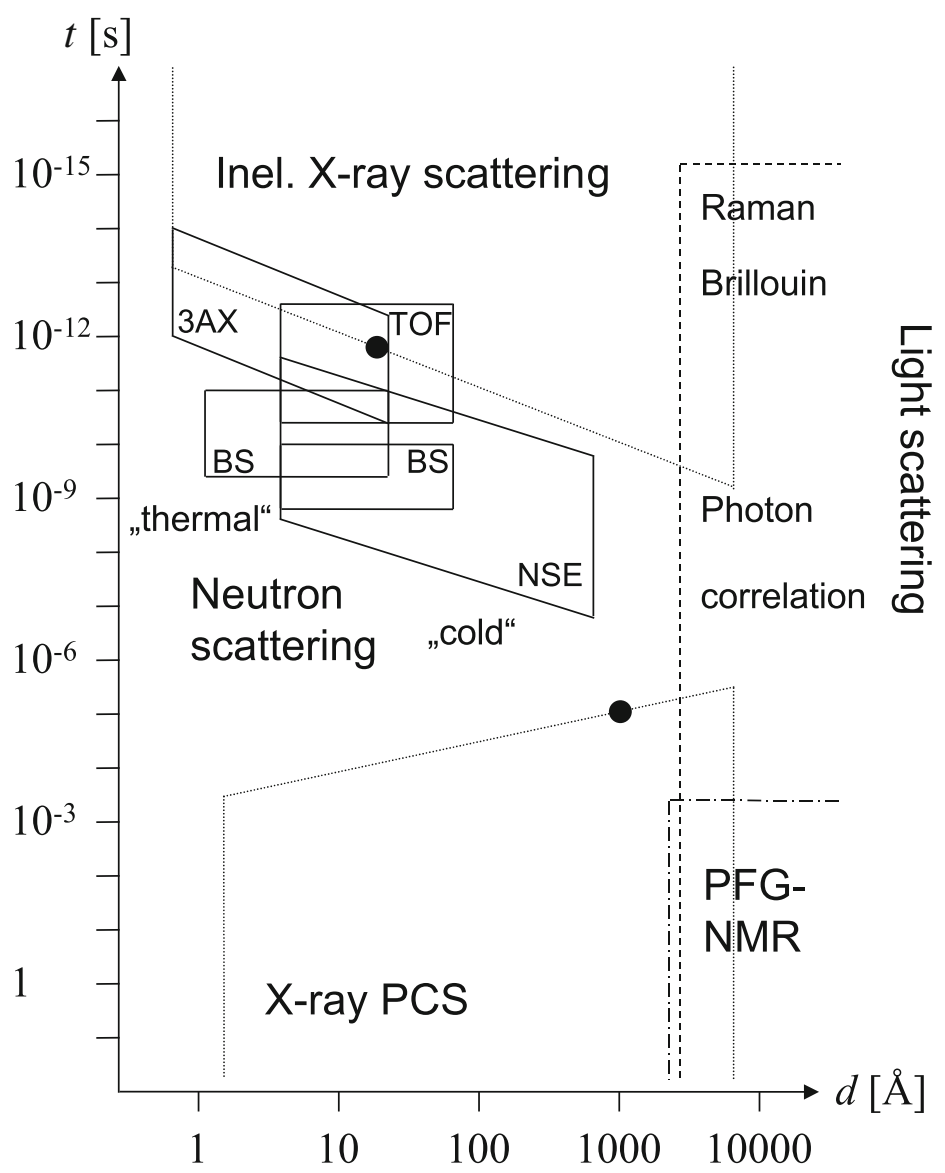

Figure 1. Vue d'ensemble des méthodes de diffusion dynamique: Les polygones solides indiquent les échelles de temps et d'espace pour lesquelles l'observation par la diffusion inélastique est possible, les lignes en pointillées (tirets) correspondent à la diffusion dynamique de la lumière. Les lignes pointillées (points) indiquent les échelles potentielles pour la diffusion inéastique des rayons $\mathrm{X}$, extrapolées à partir des expériences actuelles (points). A titre de comparaison la RMN à gradient de champ pulsé est aussi incluse comme méthode non basée sur la diffusion (points-tirets).

Avant de présenter ces techniques, nous faisons d'abord un bref résumé des principes fondamentaux de la diffusion inélastique de neutrons [1-3]. La quantité de base mesurée lors d'une expérience est la section efficace double différentielle :

$$
\frac{\partial^{2} \sigma}{\partial \Omega \partial \omega}=N \frac{k_{\mathrm{f}}}{k_{\mathrm{i}}}\left(\left(\left\langle b^{2}\right\rangle-\langle b\rangle^{2}\right) S_{\mathrm{inc}}(\mathbf{q}, \omega)+\langle b\rangle^{2} S_{\mathrm{coh}}(\mathbf{q}, \omega)\right)
$$

qui, lorsqu'elle est multipliée par le flux de neutrons incident, donne le nombre de neutrons diffusés dans un élément d'angle solide $d \Omega$ avec une énergie de transfert $\hbar \omega$. La variable est la longueur de diffusion qui est caractéristique de chaque noyau et de l'orientation relative du spin du noyau et du neutron incident. Il arrive souvent que plusieurs isotopes d'un même noyau soient présents dans l'échantillon, et aussi que les orientations de spin soient distribuées aléatoirement. Dans les deux cas $\left\langle b^{2}\right\rangle \neq\langle b\rangle^{2}$, et le premier terme, la diffusion incohérente, ne disparaît pas.

Les fonctions de diffusion $S_{\text {coh }}(\mathbf{q}, \omega)$ et $S_{\text {inc }}(\mathbf{q}, \omega)$ donnent un accès direct aux mouvements microscopiques des noyaux. La fonction de diffusion cohérente est reliée par une double transformée de 


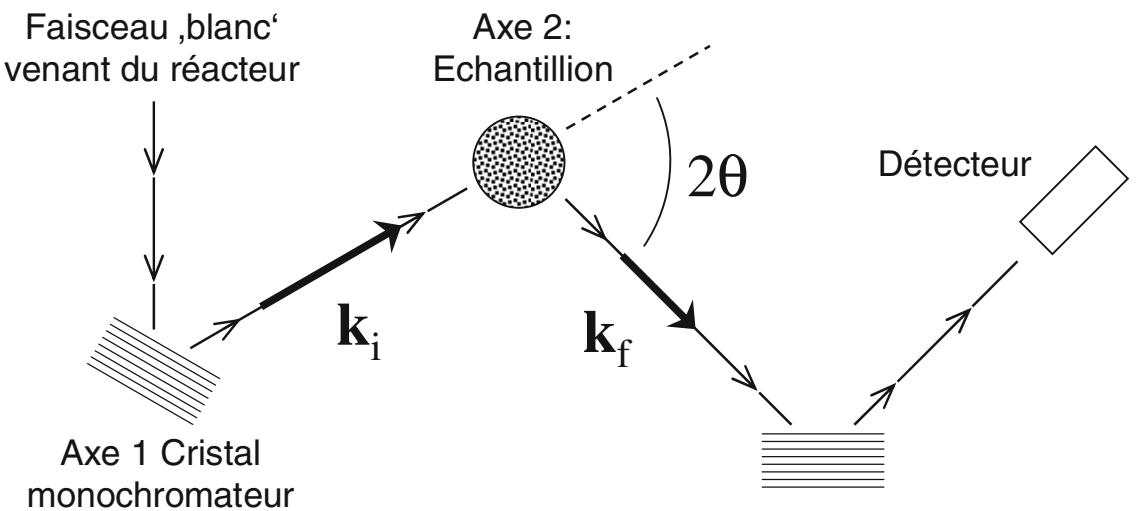
monochromateur

\section{Axe 3: Cristal} analyseur

Figure 2. Montage schématique d'un spectromètre trois-axes.

Fourier dans l'espace et le temps

$$
S_{\mathrm{coh}}(\mathbf{q}, \omega)=\frac{1}{2 \pi} \int \mathrm{d}^{3} r \int \mathrm{d} t \exp (\mathrm{i}(\mathbf{q} \cdot \mathbf{r}-\omega t)) G(\mathbf{r}, t)
$$

à la fonction de corrélation de densités

$$
G(\mathbf{r}, t)=\frac{1}{\rho_{0}}\langle\rho(\mathbf{0}, 0) \rho(\mathbf{r}, t)\rangle .
$$

Dans une description classique cette dernière fonction peut être décrite par les positions des atomes :

$$
G(\mathbf{r}, t)=\frac{1}{N}\left\langle\int \mathrm{d}^{3} \mathbf{r}^{\prime} \sum_{i, j} \delta\left(\mathbf{r}-\mathbf{r}^{\prime}+\mathbf{r}_{j}(t)\right) \delta\left(\mathbf{r}^{\prime}-\mathbf{r}_{i}(0)\right)\right\rangle .
$$

C'est pourquoi $G(\mathbf{r}, t)$ est classiquement interprétée comme la densité de probabilité de trouver un atome au temps $t$ à une distance $\mathbf{r}$ de la position d'un atome au temps 0 . Dans cette fonction de corrélation il n'importe pas que les deux atomes considérés soient les mêmes ou non. En substituant (4) dans (2), on obtient une représentation directe de la fonction de diffusion cohérente en termes de mouvements atomiques :

$$
S_{\mathrm{coh}}(\mathbf{q}, \omega)=\frac{1}{2 \pi N} \int \mathrm{d} t\left\langle\sum_{i, j} \exp \left(\mathrm{i}\left(\mathbf{q} \cdot\left(\mathbf{r}_{j}(t)-\mathbf{r}_{i}(0)\right)-\omega t\right)\right)\right\rangle .
$$

La fonction de diffusion incohérente est reliée par

$$
S_{\text {inc }}(\mathbf{q}, \omega)=\frac{1}{2 \pi} \int \mathrm{d}^{3} r \int \mathrm{d} t \exp (\mathrm{i}(\mathbf{q} \cdot \mathbf{r}-\omega t)) G_{\mathrm{s}}(\mathbf{r}, t)
$$

à la fonction d'autocorrélation

$$
G_{\mathrm{s}}(\mathbf{r}, t)=\frac{1}{N}\left\langle\int \mathrm{d}^{3} \mathbf{r}^{\prime} \sum_{i} \delta\left(\mathbf{r}-\mathbf{r}^{\prime}+\mathbf{r}_{i}(t)\right) \delta\left(\mathbf{r}^{\prime}-\mathbf{r}_{i}(0)\right)\right\rangle .
$$

Cette fonction est la densité de probabilité de trouver un atome au temps $t$ à la distance $\mathbf{r}$ de sa propre position au temps 0 . Il apparaît alors clairement que la fonction de diffusion incohérente est liée aux 
Tableau 1. Sections efficaces de diffusion de neutrons des éléments communs dans les matériaux organiques $\left(1\right.$ barn $\left.=10^{-28} \mathrm{~m}^{2}\right)$.

\begin{tabular}{ccc}
\hline Element & $\sigma_{\mathrm{coh}}=4 \pi\langle b\rangle^{2}[\mathrm{barn}]$ & $\sigma_{\text {inc }}=4 \pi\left(\left\langle b^{2}\right\rangle-\langle b\rangle^{2}\right)[$ barn $]$ \\
\hline $\mathrm{C}$ & 5.551 & 0.001 \\
$\mathrm{H}$ & 1.7568 & $\mathbf{8 0 . 2 6}$ \\
$\mathrm{D}$ & 5.592 & 2.05 \\
$\mathrm{O}$ & 4.232 & 0.0008 \\
$\mathrm{~N}$ & 11.01 & 0.5 \\
$\mathrm{~S}$ & 1.0186 & 0.007 \\
\hline
\end{tabular}

déplacements des atomes:

$$
S_{\text {inc }}(\mathbf{q}, \omega)=\frac{1}{2 \pi N} \int \mathrm{d} t\left\langle\sum_{i} \exp \left(\mathrm{i}\left(\mathbf{q} \cdot \Delta \mathbf{r}_{i}(t)-\omega t\right)\right)\right\rangle .
$$

Il est évident que cette fonction est plus facile à interpréter que son homologue cohérente. Ceci est particulièrement vrai lorsque l'approximation Gaussienne est valide, e.g., dans la limite des petits $q$, pour de la diffusion simple ou des mouvements dans des potentiels harmoniques. Dans ce cas, (8) se réduit à :

$$
S_{\text {inc }}(q, \omega)=\frac{1}{2 \pi} \int \mathrm{d} t \exp (-\mathrm{i} \omega t) \exp \left(-\frac{\left\langle\Delta r^{2}(t)\right\rangle q^{2}}{6}\right) .
$$

Ici, la moyenne doit être considérée comme une moyenne d'ensemble ainsi que sur tous les atomes. Ainsi, dans l'approximation Gaussienne, la fonction de diffusion incohérente dépend seulement du déplacement carré moyen des atomes, $\left\langle\Delta r^{2}(t)\right\rangle$.

Le Tableau 1 présente les préfacteurs respectifs des fonctions incohérentes et cohérentes dans (1), i.e., les sections efficaces de diffusion incohérente et cohérente, pour les éléments les plus courants des matériaux organiques. Il apparaît que pour des systèmes contenant de l'hydrogène, la diffusion incohérente des noyaux domine celle des autres d'un ordre de grandeur. C'est pourquoi dans ce cas, la diffusion inélastique de neutrons mesure essentiellement l'autocorrélation des mouvements de l'hydrogène. Si l'on veut mesurer la diffusion cohérente, il est nécessaire de remplacer l'hydrogène par du deutérium. Cependant, même pour un échantillon complétement deutéré, une contribution significative de la diffusion incohérente devra être prise en compte.

Une séparation expérimentale de la diffusion cohérente et incohérente est possible si elle est uniquement due à un désordre de spin (pas isotopique). C'est justement le cas pour l'hydrogène et le deutérium. De manière générale la diffusion incohérente de l'hydrogène mène à un renversement des spins (spin flip) des neutrons diffusés avec une probabilité de $2 / 3$. Il est possible de calculer les fractions de diffusion cohérente et incohérente à partir d'une analyse de polarisation. Bien que certains instruments soient dotés d'une telle possibilité d'analyse, celle-ci est rarement utilisée en raison des très faibles taux de comptages obtenus.

\subsection{Spectromètre à Temps de Vol}

Le désavantage principal d'un spectromètre 3-axes est qu'il ne permet d'observer qu'un couple $(q, \omega)$ à la fois. Alors que ceci est acceptable pour des échantillons pour lesquels toute la diffusion est concentrée dans des pics de Bragg, il est préférable de pouvoir effectuer une observation simultanée sur une gamme de $q$ et de transfert d'énergie $\hbar \omega$ lorsqu'on s'intéresse à des systèmes présentant de la diffusion diffuse. Ceci est réalisé en entourant la position échantillon d'un réseau de détecteurs. De plus, l'énergie des neutrons diffusés est mesurée par leur temps de vol: un chopper placé dans le faisceau incident définit le temps de départ et, à partir du temps que mets un neutron pour arriver au détecteur, on peut calculer 


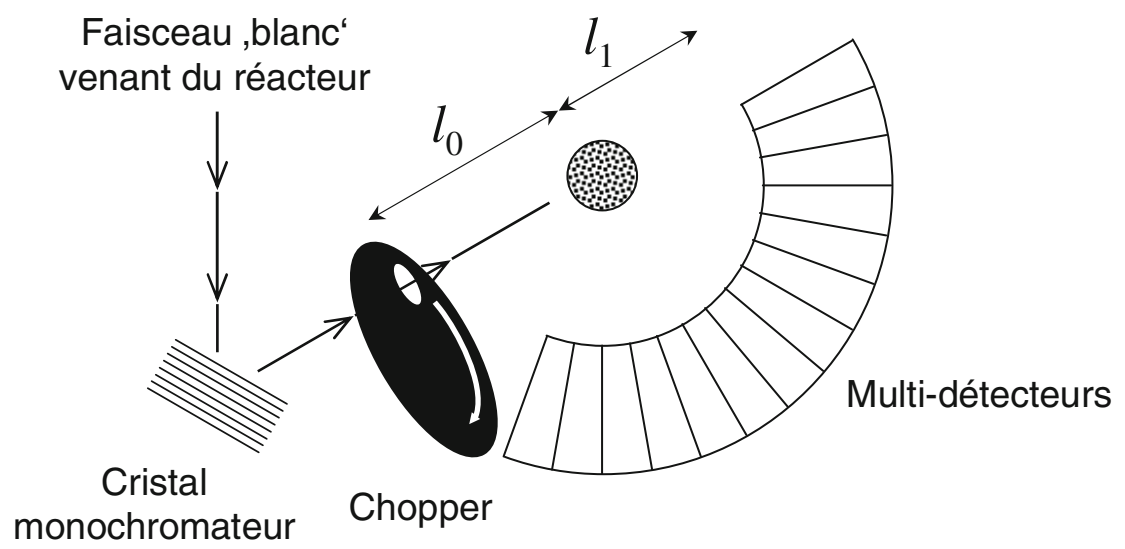

Figure 3. Montage schématique d'un spectromètre de temps-de-vol avec un cristal monochromateur.

sa vitesse, et donc son énergie de transfert par :

$$
\hbar \omega=\left(\frac{l_{1}^{2}}{\left(l_{0}-\sqrt{E_{\mathrm{i}} / m_{\mathrm{n}}} t_{\text {flight }}\right)^{2}}-1\right) E_{\mathrm{i}} .
$$

La monochromatisation du faisceau incident peut être réalisé soit par la réflexion de Bragg d'un cristal, soit par une succession de choppers phasés de telle sorte qu'ils ne transmettent qu'une seule longueur d'onde. Le premier principe permet usuellement d'atteindre des intensités plus importantes alors que le second est plus flexible pour la sélection de $E_{\mathrm{i}}$ et permet d'atteindre une meilleure résolution en énergie.

Pour l'interprétation des résultats de temps de vol, il est important de savoir que les résultats bruts, $n\left(\theta, t_{\mathrm{vol}}\right)$, le nombre de neutrons mesurés en fonction de l'angle et du temps de vol, ont une apparence très fortement différente de celle de la fonction de diffusion $S(q, \omega)$. La raison principale vient du fait que la relation (10) est non-linéaire et conduit à donner plus de poids aux canaux des temps courts qui contiennent la part du spectre contenant les $\hbar \omega$ élevés. De plus, le spectre pour un seul détecteur à un angle $\theta$ ne correspond pas à un $q$ constant mais à une ligne courbe dans le plan $(q, \omega)$ décrit par

$$
q=\frac{\sqrt{2 m_{\mathrm{n}}}}{\hbar} \sqrt{2 E_{\mathrm{i}}+\hbar \omega-2 \sqrt{E_{\mathrm{i}}\left(E_{\mathrm{i}}+\hbar \omega\right)} \cos 2 \theta} .
$$

La Figure 5 présente l'intégralité de la gamme couverte pour un instrument typique (IN6 à l'ILL, Grenoble) en utilisant des neutrons incidents de $5.1 \AA$ et des détecteurs dans la gammme angulaire $10^{\circ} \ldots 115^{\circ}$.

Le tableau 2 présente quelques instruments de TdV avec leurs spécifications. En fonction de la longueur d'onde incidente désirée, les instruments sont construits soit en utilisant directement les neutrons provenant du modérateur du réacteur (neutrons thermiques, $\lambda_{\max } \approx 1.8 \AA$ ), ou d'une source froide où une modération additionnelle des neutrons est effectuée, en général par de l'hydrogène liquide $\left(\lambda_{\max } \approx 4 \AA\right.$ ). Les neutrons thermiques permettent d'accéder à une plus grande gamme de $q$ alors que les neutrons froids donnent une meilleure résolution en énergie. C'est pourquoi le choix de l'instrument dépend du système que l'on désire observer. En général, les instruments utilisant des "neutrons froids" sont préférés pour les expériences d'INS sur les systèmes de la matière molle.

Une variante du spectromètre $\mathrm{TdV}$ existe sur les sources à spallation, le spectromètre de TdV inversé. Puisque les neutrons sont produits par bouffées sur une source à spallation, on peut utiliser leur moment de création pour démarrer l'horloge du temps de vol et il n'y a en principe pas besoin de chopper. De cette façon tous les neutrons peuvent être utilisés, au contraire des spectromètres de TdV conventionnels 

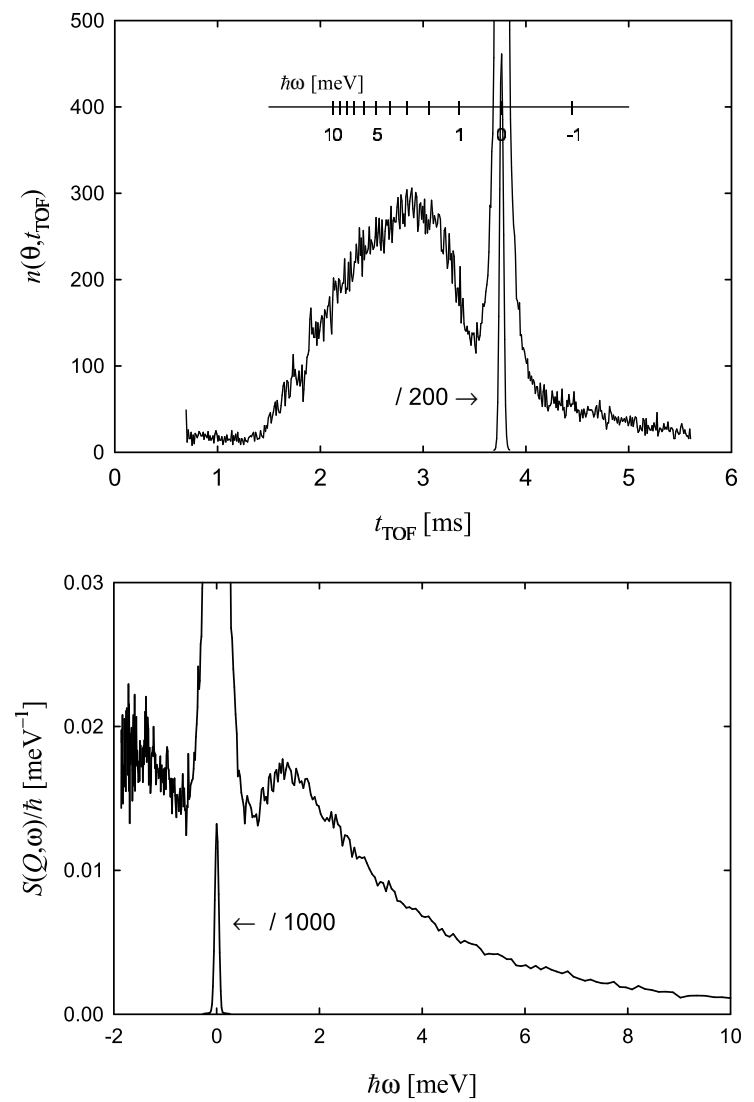

Figure 4. Haut: Résultats bruts d'un spectromètre TdV: nombre de neutrons dans un canal de $10 \mu$ s pendant une durée d'enregistrement d'une heure. l'échantillon est un liquide formant un verre confiné mésoscopiquement. l'axe flottant non linéaire indique les transferts d'énergie calculés par l'équation (10). Du fait de l'intensité élevée de la partie élastique, la partie du spectre lui correspondant a été réduite d'un facteur 200. Bas : Les mêmes données de TdV converties en $S(q, \omega)$, la ligne élastique réduite d'un facteur 1000. Les modes vibrationnels caractéristiques du matériau à $\hbar \omega \approx 1.7 \mathrm{meV}\left(14 \mathrm{~cm}^{-1}\right)$ ne deviennent visibles qu' après transformation.

Tableau 2. Spécifications basiques de quelques spectromètres de TdV représentatifs. Types d'instruments: TXthermal, crystal; CX-cold, crystal; CC-cold, chopper; I-inverse. Le $q$ maximal et la résolution en énergie $\Delta \hbar \omega$ dépendent de la longueur d'onde incidente; les limites supérieures de leurs gammes correspondent à la limite inférieure de la longueur d'onde incidente $\lambda_{\mathrm{i}}$ et vice versa.

\begin{tabular}{lcccc}
\hline Instrument & Type & $\lambda_{\mathrm{i}}[\AA]$ & $q_{\max }\left[\AA^{-1}\right]$ & $\Delta \hbar \omega[\mathrm{meV}]$ \\
\hline IN4 (ILL) & TX & $0.8-4$ & $3-14$ & $0.25-6$ \\
IN5 (ILL) & CC & $2-15$ & $0.8-5.7$ & $0.01-6$ \\
IN6 (ILL) & CX & $4.1-5.9$ & $1.8-2.6$ & $0.05-0.17$ \\
NEAT (HMI) & CC & $1.8-19$ & $0.6-6.5$ & $0.006-5$ \\
IRIS (RAL) & I & $\lambda_{\mathrm{f}}=6.7-20$ & $0.6-1.9$ & $0.001-0.015$ \\
\hline
\end{tabular}

qui n'en utilisent que quelques pourcents. l'énergie incidente est ainsi mesurée par le temps de vol et l'énergie finale est gardée constant par un ensemble fixe de cristaux analyseurs ("géométrie inverse"). En plaçant ces cristaux à une position proche de celle de la rétrodiffusion (voir la section suivante pour plus de détails), il est possible d'atteindre une excellente résolution en énergie, proche de celle des vrais spectromètres de rétrodiffusion. En conséquence directe de cette bonne fonction de résolution, les taux 


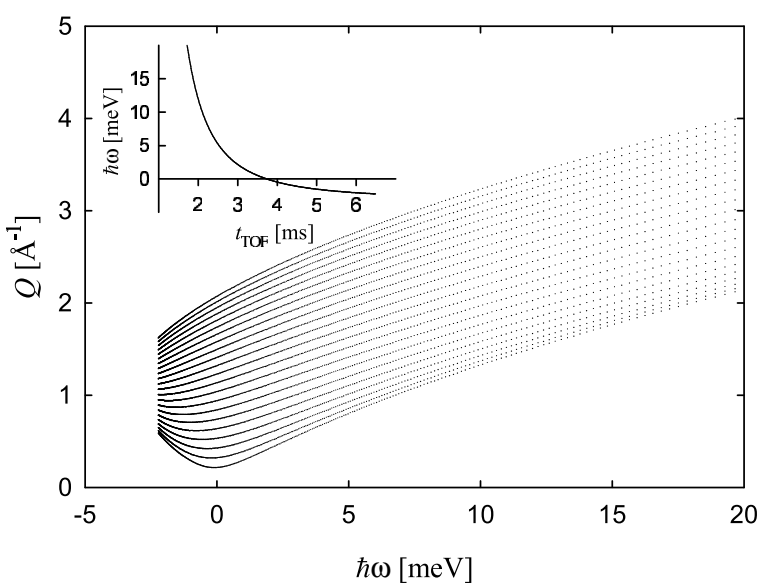

Figure 5. Gamme de points $S(q, \omega)$ accessible par un spectromètre de TdV typique, IN6 à l'ILL, Grenoble: longueur d'onde incidente $\lambda_{\mathrm{i}}=5.1 \AA$, chemin de vol secondaire $247 \mathrm{~cm}$, détecteurs dans la gamme angulaire $10^{\circ} \ldots 115^{\circ}$. Les points correspondent aux canaux de temps de vol espacés de $10 \mu$ s et les détecteurs tous les $5^{\circ}$ (l'angle augmente de la courbe du bas vers celle du haut). L'insert montre le transfert d'énergie vs. la relation $\mathrm{TdV}(10)$ pour ces conditions.

de comptage sont bas, en particulier sur les sources à spallation actuelles qui ont des taux de production de neutrons significativement plus bas que les réacteurs.

\subsection{Spectromètre de rétrodiffusion (Backscattering)}

Un problème récurrent des études sur les systèmes de matière molle est que les processus sont trop lents pour être observés. Sans recourir à des configurations extrêmes qui conduisent à une perte d'intensité, la résolution en énergie des spectromètres $\mathrm{TdV}$ est limitée à environ $10 \mu \mathrm{eV}$, ce qui correspond à une échelle de temps maximale de 200 ps. Ce n'est souvent pas suffisant pour sonder les mouvements à grande échelle des polymères ou les relaxations associées à la transition vitreuse. C'est pourquoi des instruments avec une meilleure résolution en énergie sont souvent nécessaires : le spectromètre de rétrodiffusion (backscattering BS) et le spectromètre à écho de spin (NSE).

La résolution en énergie est limitée par la sélectivité du cristal monochromateur ${ }^{1}$. Si des cristaux parfaits sont utilisés, la répartition des longueurs d'ondes sélectionnées $\Delta \lambda / \lambda$ est déterminée par la divergence angulaire des neutrons réfléchis. En dérivant la condition de Bragg, on obtient :

$$
\Delta \lambda / \lambda=\cot \theta \cdot \Delta \theta \text {. }
$$

Cette expression devient nulle pour $2 \theta=180^{\circ}$. En pratique ceci signifie que la dispersion devient minimale si le faisceau de neutrons est réfléchi de $180^{\circ}$, i.e., en condition de rétrodiffusion.

La Figure 6 présente schématiquement un instrument basé sur ce principe. Le premier cristal dans le faisceau est simplement un déflecteur avec une faible sélectivité en longueur d'onde. La monochromatisation effective a lieu sur la seconde réflexion par le cristal en position de rétrodiffusion. Les neutrons sont ensuite diffusés par l'échantillon qui est entouré d'analyseurs placés sur une surface sphérique. Ils sont alors de nouveau diffusés en condition de rétrodiffusion. Les neutrons réfléchis passent encore une fois à travers l'échantillon puis atteignent finalement le détecteur.

\footnotetext{
1 Pour les spectromètres à choppers la limite est donnée par la longueur du pulse qui peut être, sur le principe, arbitrairement petite. Mais, puisque l'intensité mesurée décroît de façon quadratique avec la durée du pulse, la résolution limite d'une expérience optimisée est en pratique du même ordre de grandeur.
} 


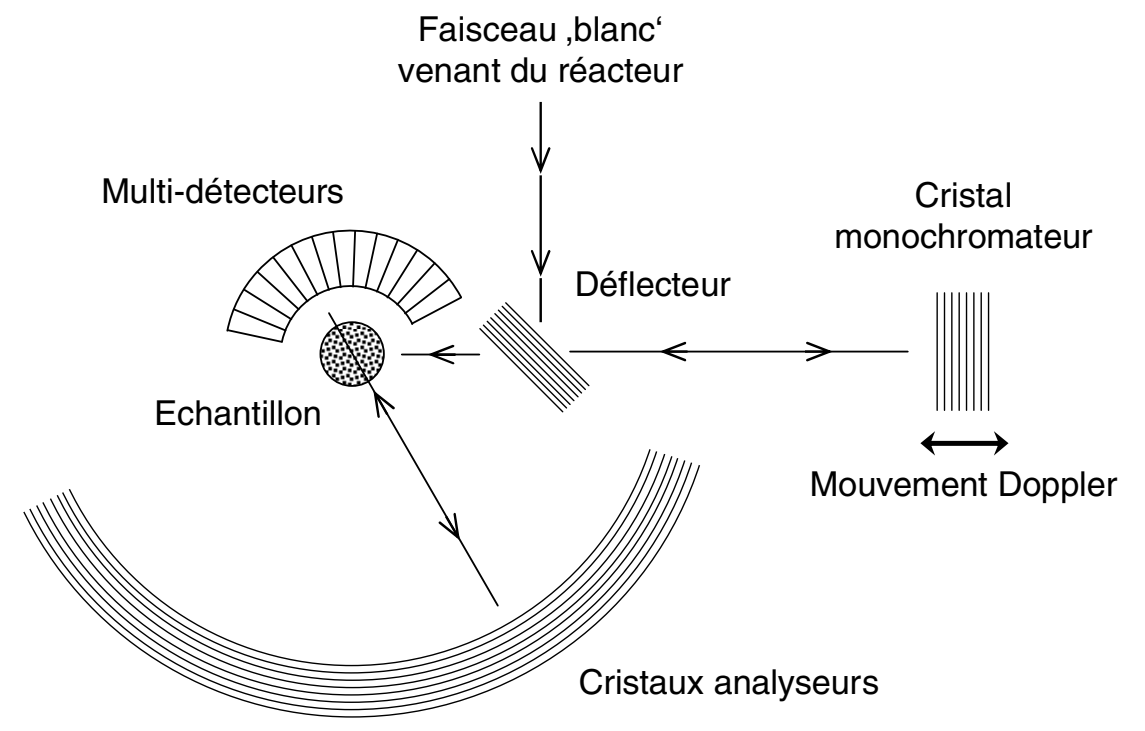

Figure 6. Montage schématique d'un spectromètre de rétrodiffusion.

Tableau 3. Spécifications basiques de quelques spectromètres caractérisitiques de rétrodiffusion de neutrons. Types d'instruments : CD-cold, Doppler monochromator; $\mathrm{CH}$-cold, heated monochromator; TH-thermal, heated monochromator. Le $q$ maximal et la résolution en énergie $\Delta \hbar \omega$ dépendent de la longueur d'onde incidente; les limites supérieures de leurs gammes correspondent à la limite inférieure de la longueur d'onde incidente $\lambda_{\mathrm{i}}$ et vice versa.

\begin{tabular}{lccccc}
\hline Instrument & Type & $\lambda[\AA]$ & $q_{\max }\left[\AA^{-1}\right]$ & $\Delta \hbar \omega[\mu \mathrm{eV}]$ & $\hbar \omega_{\max }[\mu \mathrm{eV}]$ \\
\hline IN16 (ILL) & $\mathrm{CD}$ & 6.27 & 1.9 & $0.2-1.0$ & 15 \\
IN10B (ILL) & $\mathrm{CH}$ & 6.29 & 2.0 & 1.5 & 120 \\
IN13 (ILL) & TH & 2.23 & 5.5 & 8 & 300 \\
SPHERES (JCNS) & $\mathrm{CD}$ & 6.27 & 1.8 & 0.7 & 30 \\
\hline
\end{tabular}

Il apparaît que la condition de rétrodiffusion pose des problèmes techniques à plusieurs endroits : (1) Le déflecteur ne doit pas accepter tous les neutrons, sinon le faisceau monochromatique serait de nouveau rediffusé vers la source. Ceci peut être résolu en le mettant sur un disque en rotation qui l'élimine au moment où les neutrons reviennent du monochromateur. Toutes ces mesures se font évidemment au détriment de l'intensité. (2) le deuxième passage des neutrons diffusés à travers l'échantillon entraîne une diffusion multiple et une absorption additionnelles. Les deux problèmes peuvent être résolus en abandonnant exactement la condition de rétrodiffusion, avec la conséquence que la résolution en énergie se dégrade.

Jusqu'ici il semble qu'un appareil de rétrodiffusion ne puisse observer que de la diffusion élastique $\left(E_{\mathrm{i}}=E_{\mathrm{f}}\right)$ si on utilise les mêmes cristaux pour le monochromateur et l'analyseur. Pour faire de la diffusion inélastique on doit changer soit $E_{\mathrm{i}}$ soit $E_{\mathrm{f}}$. Il s'avère que c'est beaucoup plus facile pour l'énergie incidente en utilisant soit un monochromateur en mouvement (effet Doppler), soit en un monochromateur chauffée (l'expansion thermique modifiant la maille du réseau $d$ ). Cette dernière technique permet généralement des transferts d'énergie plus grands. Pour de très grands transferts d'énergie différents cristaux sont utilisés pour le monochromateur et pour l'analyseur, ce qui induit un décalage de toute la gamme de $\hbar \omega$. Le tableau 3 donne les spécifications de quelques spectromètres de rétrodiffusion caractéristiques. 


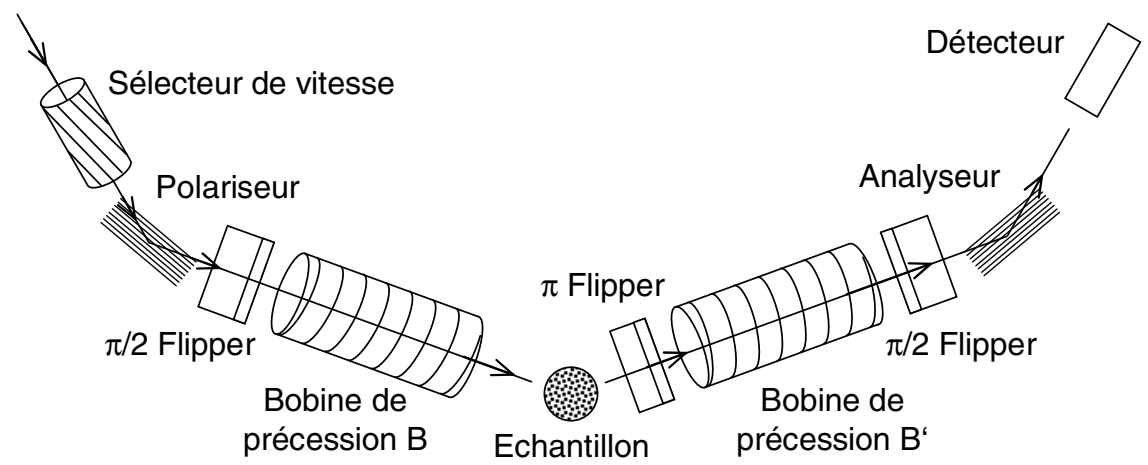

Figure 7. Montage schématique d'un spectromètre à écho de spin.

\begin{tabular}{lr}
\hline$\left(n_{x}, n_{y}, n_{z}\right)$ & dispositif neutronique \\
\hline$(0,0,1)$ & $\pi / 2$ flipper \\
$(1,0,0)$ & champ $B$ \\
$(\cos \phi, \sin \phi, 0)$ & $\pi$ flipper \\
$(\cos \phi,-\sin \phi, 0)=$ & champ $B^{\prime}$ \\
$(\cos (-\phi), \sin (-\phi), 0)$ & \\
$\left(\cos \left(\phi^{\prime}-\phi\right), \sin \left(\phi^{\prime}-\phi\right), 0\right)$ & $\pi / 2$ flipper \\
$\left(0, \sin \left(\phi-\phi^{\prime}\right), \cos \left(\phi-\phi^{\prime}\right)\right)$ & \\
\hline
\end{tabular}

\subsection{Spectromètre à echo de spin}

Pour accéder à des processus dynamiques encore plus lents en matière molle, une technique à très haute résolution est requise pour atteindre plus de 100 nanosecondes, ce qui correspond à des transferts d'énergies dans la gamme du neV. Une telle technique est fournie par les spectromètres à écho de spins de neutrons [4] qui sont capables de mesurer directement les changements d'énergie dus à la diffusion.

Ceci distingue le NSE (Neutron Spin Echo) des techniques INS conventionnelles qui procèdent en deux étapes : (1) monochromatisation du faisceau incident pour $E_{\mathrm{i}}$, (2) analyse du faisceau diffusé $\left(E_{\mathrm{f}}\right)$. Le transfert d'énergie est déterminé par différence $E_{\mathrm{f}}-E_{\mathrm{i}}$. Pour obtenir de hautes résolutions en énergie avec ces techniques, on choisit un intervalle en énergie très étroit à partir du spectre de neutrons de la source qui présente une intensité relativement basse. Les techniques conventionnelles ménent inévitablement au problème des faibles taux de comptage sur le détecteur.

Contrairement à ces méthodes, la NSE mesure les différences des vitesses individuelles des neutrons incidents et des neutrons diffusés en utilisant la précession de Larmor du spin du neutron dans un champ magnétique. Le vecteur du spin du neutron joue le rôle de l'aiguille d'une horloge interne liée à chaque neutron et lie le résultat de la mesure de la vitesse au neutron lui-même. Il est ainsi possible de comparer les vitesses avant et après diffusion sur un seul et même neutron et une mesure directe de la différence de vitesse devient possible. La résolution en énergie est alors découplée de la monochromatisation du faisceau incident. Des résolutions en énergie de l'ordre de $10^{-5}$ peuvent être obtenues à partir d'un spectre de neutrons ayant une bande passante de $20 \%$. 
Le mouvement de la polarisation des spins du neutron $\mathbf{P}(t)$ - qui est la valeur attendue en mécanique quantique du spin du neutron - est décrit par l'équation de Bloch

$$
\frac{\mathrm{d} \mathbf{P}}{\mathrm{d} t}=\frac{\gamma \mu}{\hbar}(\mathbf{P} \times \mathbf{B})
$$

ou $\gamma$ est le rapport gyromagnétique $(\gamma=-3.82)$ du neutron, $\mu$ le magnéton nucléaire et $\mathbf{B}$ le champ magnétique. La manipulation de la polarisation du neutron par des champs externes est basée sur cette équation (13). En particulier, si un neutron de longueur d'onde $\lambda$ est exposé à un champ magnétique $B$ sur une longueur $l$ de sa distance de vol, son spin est soumis à une rotation de :

$$
\phi=\left(\frac{2 \pi|\gamma| \mu \lambda m_{\mathrm{n}}}{h^{2}}\right) B l
$$

Le principe de base d'un spectromètre à écho de spins est présenté sur la figure 7. Un monochromateur mécanique sélectionne dans le faisceau de neutrons primaire un intervalle de longueur d'ondes de largeur 10-20\%. Dans les trajectoires de vol primaire et secondaire de l'instrument, des champs de précession $B$ and $B^{\prime}$ parallèles aux trajectoires respectives sont générés par des bobines cylindriques. Avant d'entrer dans la première partie, le faisceau de neutron est polarisé vers l'avant dans la direction de propagation ${ }^{2}$. Premièrement, un flipper $\pi / 2$ fait tourner la polarisation dans la direction $x$ perpendiculaire à la direction de propagation $(z)$. Ceci est réalisé en exposant les neutrons à un champ magnétique très bien défini durant un temps donné par leur vitesse et l'épaisseur de la bobine plate (bobine de Mezei). En commençant par cette condition parfaitement définie les neutrons commencent leur précession dans le champ $B$. Après avoir été diffusés par l'échantillon les neutrons passent à travers un flipper $\pi$ et à travers le deuxième champ de précession $B^{\prime}$. Finalement, les neutrons passent à travers une autre bobine $\pi / 2$ qui, sous certaines conditions, restaure leur polarisation initiale parallèlement à la direction de vol. Pour comprendre ce qu'est cette condition, il faut retracer les changements du vecteur spin ( $z$ indiquant toujours la direction parallèle à la direction de propagation des neutrons) :

Au total, le spin a subi une rotation de $\phi-\phi^{\prime}$ autour de l'axe $x$ lorsque le neutron passe à travers le spectromètre. Ceci signifie que la polarisation finale est identique à la polarisation incidente si $\phi=\phi^{\prime}$ $(+2 \pi n)$, en particulier lorsque $\lambda_{\mathrm{i}}=\lambda_{\mathrm{f}}$ (diffusion élastique) et $\int_{0}^{l} B \mathrm{~d} z=\int_{0}^{l^{\prime}} B^{\prime} \mathrm{d} z$ (pour des champs homogènes : $B l=B^{\prime} l^{\prime}$ ), comme le montre l'équation (14). Cette condition est appelée "écho de spin" et est indépendante des vitesses individuelles des neutrons parce que $\phi-\phi^{\prime}$ est déterminé par leur seule différence.

Si on s'écarte de la condition d'écho de spin la probabilité pour un neutron donné d'atteindre le détecteur est réduite de $\cos \left(\phi^{\prime}-\phi\right)$ du fait de l'analyseur de polarisation. Si l'on garde la symétrie de l'instrument, $B l=B^{\prime} l^{\prime}$, mais que l'on considère la diffusion inélastique, le décalage de l'angle de précession peut être approximé par :

$$
\begin{aligned}
\phi^{\prime}-\phi & =\left(\frac{2 \pi|\gamma| \mu m_{\mathrm{n}}}{h^{2}}\right) B l\left(\lambda_{\mathrm{f}}-\lambda_{\mathrm{i}}\right) \\
& \approx \underbrace{\frac{|\gamma| \mu m_{\mathrm{n}}{ }^{2} \lambda^{3} B l}{h^{3}}}_{=t_{\mathrm{NSE}}(B)} \omega
\end{aligned}
$$

pour les petits transferts d'énergie pour lesquels $\Delta \lambda \approx \hbar \omega / \frac{\mathrm{d} E}{\mathrm{~d} \lambda}$ peut être uilisé. Puisque le transfert d'énergie pour la diffusion inélastique n'est pas fixé mais distribué tel que le détermine la fonction de diffusion $S(q, \omega)$ il faut faire une moyenne du facteur $\cos \left(\phi^{\prime}-\phi\right)$ pondéré de $S(q, \omega)$ pour obtenir la

\footnotetext{
2 Ceci est réalisé par un "supermiroir polarisant" qui ne réfléchit que les neutrons ayant ce spin - similaire au prisme de Nicol en optique.
} 
réduction du taux de comptage sur le détecteur, la polarisation effective

$$
P\left(q, t_{\mathrm{NSE}}\right)=\frac{\int_{-\infty}^{\infty} S(q, \omega) \cos \left(\omega t_{\mathrm{NSE}}\right) \mathrm{d} \omega}{\int_{-\infty}^{\infty} S(q, \omega) \mathrm{d} \omega} .
$$

Premièrement, il faut noter que, dans cette expression, $S(q, \omega)$ est usuellement la fonction de diffusion cohérente. En principe, des arguments similaires peuvent être utilisés pour la diffusion incohérente parce qu'une fraction bien définie des neutrons change son spin. Ceci conduit à un "écho négatif" parce que la majorité des neutrons inversent leur polarisation. Mais cet effet est plus difficile à observer parce que cet effet est seulement partiel (e.g. 2/3 des noyaux d'hydrogène). La spectroscopie NSE n'a pu être appliquée avec succès que récemment à des échantillons diffusant de façon incohérente.

Deuxièmement, parmi les transformées de Fourier de l'équation (2), l'expression (16) renverse celle du temps et se retrouve ainsi entre la fonction microscopique $G(\mathbf{r}, t)$ et l'observable $S(\mathbf{q}, \omega)$. Pour cette raison $^{3}$

$$
\begin{aligned}
I(\mathbf{q}, t) & =\int_{-\infty}^{\infty} S(\mathbf{q}, \omega) \exp (\mathrm{i} \omega t) \mathrm{d} \omega \\
& =\int \mathrm{d}^{3} r \exp (\mathrm{i}(\mathbf{q} \cdot \mathbf{r})) G(\mathbf{r}, t)
\end{aligned}
$$

est appelée la fonction intermédiaire de diffusion and le résultat d'une expérience de NSE

$$
P\left(q, t_{\mathrm{NSE}}(B)\right)=I\left(q, t_{\mathrm{NSE}}(B)\right) / I(q, 0)
$$

est la fonction intermédiaire de diffusion normalisée. Cette fonction est souvent plus facile à comprendre et plus simple à interpréter que la fonction de diffusion dépendant de la fréquence.

Afin d'estimer les temps de Fourier typiques $t_{\text {NSE }}$ qu'il est possible d'atteindre par NSE, nous considérons les champs maximum de $B=B^{\prime}=500$ Gauss dans les bobines de précession de longueur $l=l^{\prime}=2 \mathrm{~m}$ pour une longueur d'onde $\lambda=8 \AA$. Il résulte de (15) qu'un temps d'environ $10 \mathrm{~ns}$ peut être atteint.

De cette équation il apparaît aussi clairement que la façon la plus efficace d'augmenter ce temps est d'utiliser des longueurs d'onde plus grandes parce $\lambda$ varie à la puissance trois. Ceci réduit alors la gamme de $q$ accessible, ce qui constitue un défaut pour les matériaux composés de petites molécules mais pas pour les propriétés à grande échelle des polymères qui peuvent être observées à petits $q$ de toutes façons.

Des spectromètres typiques de NSE avec leurs spécifications sont listés dans le tableau 4. Les spectromètres de NSE sont des instruments très flexibles souvent utilisés avec des configurations différentes et seules les configurations "standard" ont été incluses ici. Comme caractéristiques particulières qui peuvent être mentionnées, IN11 et SPAN ont des réseaux de détecteurs à une dimension qui couvrent respectivement $60^{\circ}$ et $150^{\circ}$ degrés, permettant l'observation simultanée à plusieurs valeurs de $q$. Les instruments IN15 et J-NSE ont des réseaux de détecteurs à deux dimensions qui peuvent être utilisées pour des études anisotropes mais couvrent une gamme angulaire plus petite. IN15 utilise un miroir focalisant pour augmenter le flux qui serait sinon très faible du fait de la longueur des bobines de précession.

\section{DYNAMIQUE DES POLYMÈRES}

Comme exemple de dynamique en matière molle, la dynamique microscopique des polymères sera présentée. Les polymères présentent des propriétés viscoélastiques et de transport inhabituelles qui

\footnotetext{
${ }^{3}$ La transformée de Fourier exponentielle et la transformée de Fourier cosinus (16) sont identiques dans la limite classique si $S(\mathbf{q}, \omega)$ est symétrique et $I(\mathbf{q}, t)$ une fonction réelle.
} 
Tableau 4. Configurations standards de quelques spectromètres à écho de spin représentatifs. Le $q$ maximal et le temps de Fourier maximal $t_{\max }$ dépendent de la longueur d'onde incidente; la valeur limite la plus haute de la gamme de $q$ et la valeur limite basse de $t_{\max }$ correspondent à la valeur basse limite de la longueur d'onde incidente $\lambda$ et vice versa.

\begin{tabular}{lccc}
\hline Instrument & $\lambda[\AA ̊]$ & $q_{\max }\left[\AA^{-1}\right]$ & $t_{\max }[\mathrm{ns}]$ \\
\hline IN11 (ILL) & $4.5-12$ & $0.9-2.4$ & $2-45$ \\
IN15 (ILL) & $8-25$ & $0.13-0.4$ & $30-800$ \\
J-NSE (JCNS) & $4.5-16$ & $0.4-1.5$ & $10-350$ \\
SPAN (HMI) & $2.5-10$ & $1.2-4.9$ & $0.2-10$ \\
\hline
\end{tabular}

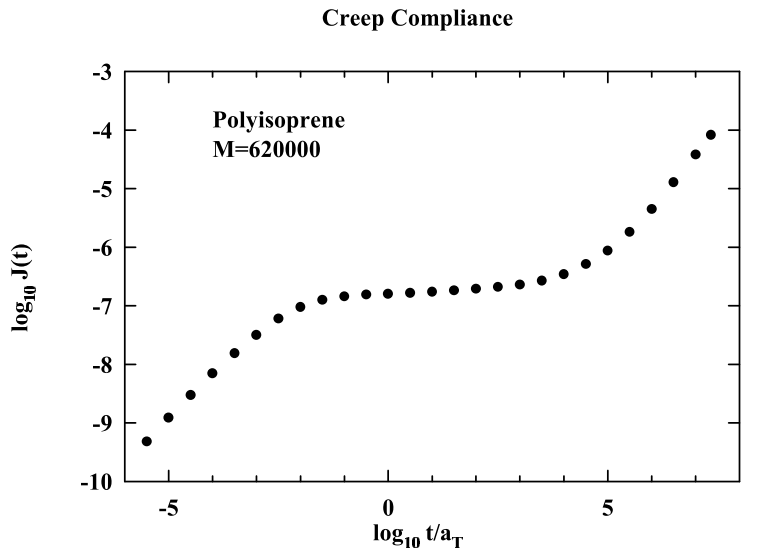

Figure 8. Compliance de cisaillement $J(t)$ du polyisoprene (poids moléculaire $M_{w}=6.2 \times 10^{5}$ ), en représentation double-logarithmique [6]. L'axe des temps a été construit en utilisant le principe de superposition tempstempérature: des résultats obtenus à différentes températures ont été renormalisés par un facteur dépendant de la température afin d'obtenir une courbe maîtresse.

déterminent dans une large mesure leurs caractéristiques de mise en forme et d'applications [5]. Ces propriétés ont également un intérêt fondamental et une compréhension à l'échelle moléculaire est nécessaire. Les polymères sont des objets mésoscopiques avec des échelles de tailles pertinentes allant de la longueur d'une simple liaison (environ $0.1 \mathrm{~nm}$ ) jusqu'a la dimension de la chaîne entière qui peut atteindre $100 \mathrm{~nm}$ ou plus. Cette variété d'échelles de taille s'accompagne d'une variété importante d'échelle de temps qui commencent dans la gamme de la picoseconde et s'étend jusqu'aux temps macroscopiques.

Un exemple typique de la viscoélasticité d'un polymère est présenté sur la figure 8 , la compliance de cisaillement $J(t)$ du polyisoprène. La compliance de cisaillement est donnée par la contrainte de cisaillement $\sigma(t)=\Delta y(t) / x$ résultant de la contrainte $\epsilon$ appliquée à $t=0$ par $J(t)=\sigma(t) / \epsilon$. Pour un milieu purement élastique, on s'attend à $J(t)=$ const en suivant la loi de Hooke, et pour un matériau visqueux $J(t)=t / \eta$ en suivant la loi de Newton. Le comportement du polymère est complexe, beaucoup plus même qu'une superposition de ces deux types de comportements. Au début l'augmentation de $J(t)$ est non-linéaire. Elle s'arrête alors pour présenter un plateau indiquant un comportement ressemblant à celui d'un ressort ou d'un caoutchouc. Le comportement visqueux $J(t) \propto t$ n'est atteint que pour des temps très longs avec une grande viscosité "terminale".

La longueur du plateau augmente avec le poids moléculaire du polymère, ce qui décale le régime visqueux vers les hautes valeurs. C'est pourquoi la viscosité augmente avec le poids moléculaire $M$. Cette augmentation peut être décrite par des lois de puissance, $\eta \propto M$ en dessous d'un certain poids moléculaire $M_{c}$ et, typiquement, $\eta \propto M^{3.4}$ au dessus (figure 9a). Une dépendance similaire est obtenue pour la constante d'auto-diffusion: $D \propto M^{-1}$ pour $M<M_{c}$, et $D \propto M^{-2}$ pour $M>M_{c}$ (figure $9 \mathrm{~b}$ ). 

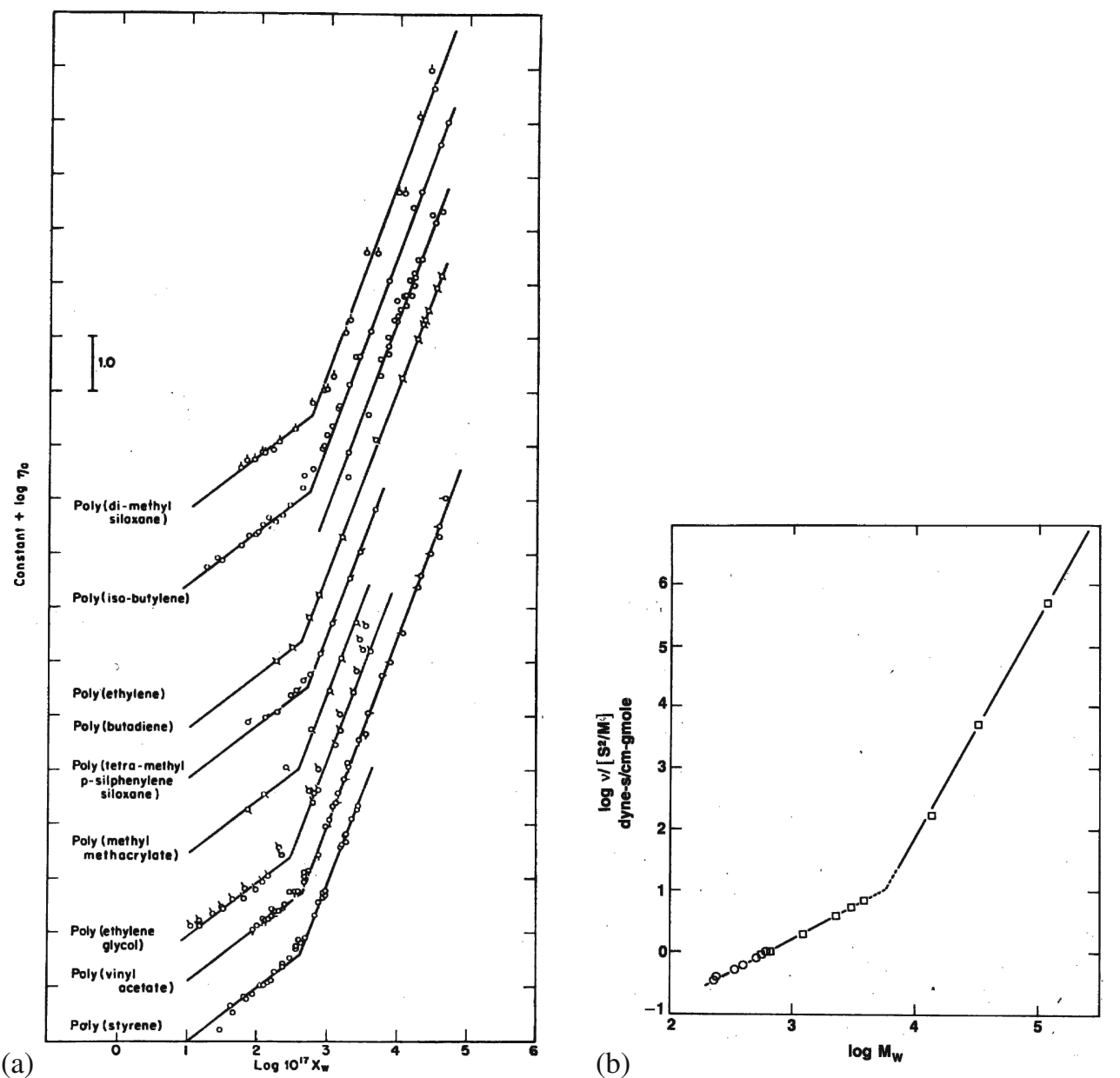

Figure 9. Dépendance du poids moléculaire de (a) la viscosité de fondus de polymères variés [7], (b) l'inverse de la constante de diffusion réciproque $D^{-1}$ du polyethylene. Reproduit avec la permission de [7] et de [8].

Le fait que les relations présentées sur la figure 9 soient applicables à pratiquement tous les polymères avec les mêmes exposants indique que la dynamique des polymères à grande échelle suit des lois universelles. La raison sous-jacente est que, sur de grandes échelles de longueur, la nature individuelle de l'unité monomérique n'est pas importante. Une description plutôt basée sur des modèles simples (e.g. billes et ressorts) est possible. De telles descriptions ont d'abord été développées dans la région pour laquelle $M<M_{c}$ puis plus tard également pour $M>M_{c}$ et seront discutées respectivement dans les paragraphes 2.1.1 and 2.1.2.

En dépit du fait que de tels concepts ont connu un succès immédiat pour la description des expériences de rhéologie, il a fallu attendre longtemps pour les confirmer sur une base moléculaire. Pour cette tâche, la diffusion inélastique des neutrons, et en particulier la technique de l'Echo de Spin de Neutrons (NSE) s'est révélée être un outil crucial pour caractériser la dynamique mésoscopique sur les échelles de longueur et de temps appropriées.

En revanche, la nature chimique du polymère devient importante sur de petites échelles de taille. Les propriétés dynamiques à cette échelle deviennent similaires à celles des liquides organiques composés de petites molécules. On trouve en particulier une transition vers un état vitreux à une température 
$T_{g}$ qui dépend fortement de la chimie (e.g. $-120^{\circ} \mathrm{C}$ Pour le polydimethylsiloxane et $+100^{\circ} \mathrm{C}$ pour le polystyrène). Des modèles utilisant des concepts universels pour décrire les systèmes sont actuellement en cours de développement mais ils ne sont pas assez aboutis pour décrire toute la dynamique des chaînes de polymères.

\subsection{Dynamique à grande échelle}

\subsubsection{Dynamique de chaîne libre (Rouse)}

En dépit de la simplicité du modèle standard pour la dynamique des chaînes courtes, le modèle de Rouse [9], il implique des calculs complexes. Les détails mathématiques peuvent être trouvés dans la littérature [10].

Ici, seules les hypothèses de base et les résultats les plus importants seront présentés. Premièrement, la chaîne est divisée en segments avec une distance bout-à-bout ayant une distribution Gaussienne. Ensuite, l'hypothèse la plus importante est qu'il n'y a pas d'interactions entre ces segments et ceux des autres chaînes ou de la même chaîne, à l'exception d'une force de friction globale et d'une force aléatoire comme on le suppose dans le calcul du mouvement Brownien d'une particule unique.

Les résultats pour les propriétés rhéologiques macroscopiques, la viscosité terminale et la constante d'auto-diffusion sont

$$
\begin{aligned}
\eta & =\frac{\zeta \ell^{2} \rho N}{36} \propto N \\
D & =\frac{k_{\mathrm{B}} T}{\zeta N} \propto N^{-1}
\end{aligned}
$$

en parfait accord avec ce qui est obtenu pour les polymères de faible poids moléculaire. $N$ est le nombre de segments, $N \propto M, \rho$ la densité du polymère, $\zeta$ le coefficient de friction, et $\ell$ la longueur moyenne d'un segment. Ces relations peuvent être justifiées par l'argument primaire que s'il n'y a pas d'autres interactions qu'une friction avec l'environnement, son effet total sur une molécule de polymère va simplement augmenter avec le nombre d'unités de frottement.

En dépit de cet argument, il reste encore à prouver que le modèle est correct au niveau moléculaire. Ici, le modèle de Rouse permet un test très strict parce qu'il prédit le facteur de structure dynamique (fonction d'auto-corrélation) :

$$
I_{\text {inc }}(q, t)=\exp \left(-\left(\frac{k_{\mathrm{B}} T \ell^{2} q^{4}}{3 \pi \zeta} t\right)^{1 / 2}\right)
$$

Des mesures de diffusion inélastique incohérente sont réalisées usuellement sur des matériaux protonés à cause de la forte section efficace incohérente de l'hydrogène (voir table 1). Mais puisque dans ce cas l'origine de la diffusion vient de l'incohérence de spin, ce qui signifie que la polarisation est partiellement perdue, des expériences de spin-écho sont difficile, voire impossible, avec des échantillons présentant une grande quantité d'hydrogène. Afin de contourner cet obstacle, on utilise une astuce chimique. Un polymère deutéré de haut poids moléculaire est synthétisé qui contient de courtes séquences hydrogénées à des positions aléatoires. Dans un tel échantillon, la diffusion provient essentiellement du contraste entre la séquence protonée et l'environnement deutéré et est donc cohérent à l'égard des diffuseurs individuels. D'un autre côté, les séquences marquées sont distribuées aléatoirement et il n'y a donc pas d'interférences constructives entre les ondes partielles provenant des 


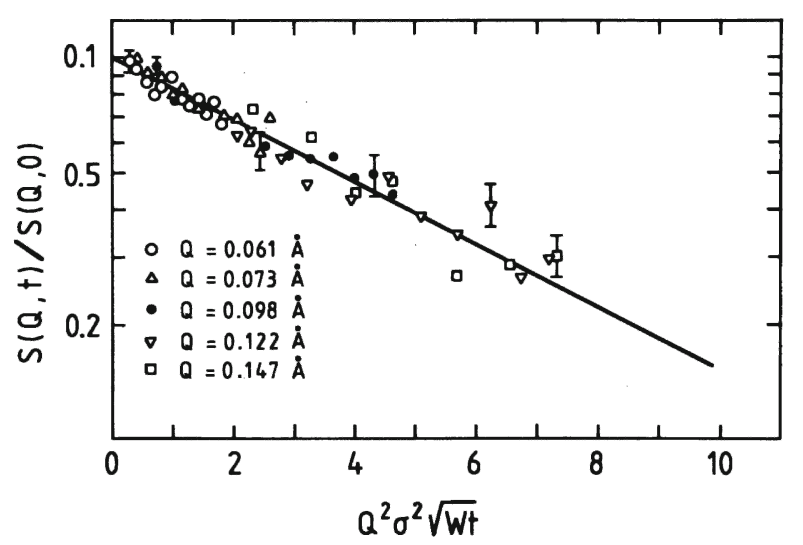

Figure 10. Spectres de spin-écho, fonction d'auto-corrélation $I_{\text {inc }}(q, t)$, mesurés sur un échantillon marqué aléatoirement de polydimethylsiloxane (poids moléculaire $M_{w}=10^{5}$ ) à $100^{\circ} \mathrm{C}$. Tracé semi-logarithmique en fonction de l'axe des temps normalisé par la variable de Rouse. La ligne continue est un ajustement avec le résultat du modèle de Rouse (21). Reproduit avec la permission de [11].

différentes séquences. Ceci signifie qu'au niveau de l'ensemble de la chaîne la diffusion est incohérente ${ }^{4}$ et l'expérience de diffusion mesure la fonction d'auto-diffusion des segments.

Sur la figure 10, les résultats de diffusion obtenus de cette façon sur du polydimethylsiloxane (PDMS) sur le spectromètre de spin-écho IN11 sont tracés en fonction de la variable d'échelle du modèle de Rouse (22)

$$
\frac{q^{2} \ell^{2}}{6} \sqrt{W t}
$$

avec $W \equiv 3 k_{\mathrm{B}} T / \ell^{2} \zeta$. Les résultats pour les différents transferts de quantités de mouvements $q$ suivent une même droite commune sur le tracé semi-logarithmique, ce qui correspond au comportement caractéristique prédit par le modèle de Rouse.

Il est aussi possible de tester le modèle de Rouse à un niveau moléculaire en utilisant la fonction intermédiaire de diffusion cohérente. Dans ce cas, on utilise un mélange de chaînes (totalement) protonées ou deutérées comme il est utilisé pour la mesure du facteur de structure de statique d'une chaîne dans une expérience de diffusion de neutrons aux petits angles. L'analyse des données est cependant plus compliquée parce qu'il n'y a pas d'expression approchée pour la fonction intermédiaire de diffusion cohérente. Cependant, puisqu'elle suit les mêmes lois d'échelle que la fonction incohérente, le test crucial est le même tracé en fonction de la variable de Rouse (22) (figure 11).

\subsubsection{Dynamique d'enchevêtrement (Reptation)}

Le comportement suivant le modèle simple de Rouse obtenu pour le PDMS de poids moléculaire d'environ $10^{5}$ est plutôt une exception qu'une règle en science des polymères. Ceci devient déjà clair sur les propriétés macroscopiques $\eta$ et $D$ qui ne suivent les prédictions du modèle de Rouse que lorsque $M<M_{c}$. A cet égard, le PDMS est un cas particulier à cause de son faible coefficient de friction $\zeta$ et

\footnotetext{
${ }^{4}$ A ce stade, il devient clair que la division de (1) entre diffusion cohérente et incohérent peut être arbitraire. Si nous considérons l'hydrogène et le deutérium comme étant isotopes du même atome nous obtenons une différence $\left\langle b^{2}\right\rangle-\langle b\rangle^{2}$ non seulement du désordre de spin mais aussi des différences de valeurs de $b$ des noyaux. Si nous considérons H et D comme étant des atomes différents (parce que dans les échantillons considérés ils sont placés - par des moyens chimiques - à des positions préférentielles) il nous faudrait étendre (1) à une expression polyatomique qui devrait alors contenir la même diffusion pour la partie cohérent, en laissant juste le désordre de spin comme incohérent.
} 


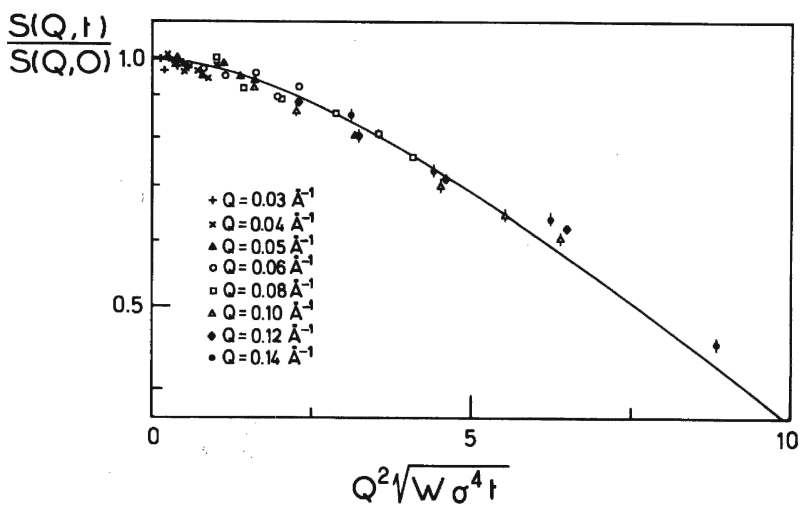

Figure 11. Spectre de spin-écho, fonction de corrélation de paire $I_{\text {coh }}(q, t)$, mesuré sur un mélange de $\mathrm{H}$ - et Dpolydimethylsiloxane (poids moléculaire $M_{w}=1.5 \times 10^{5}$ ) à $100^{\circ} \mathrm{C}$. Tracé semi-logarithmique en fonction de l'axe des temps normalisé par la variable de Rouse. La ligne continue est un ajustement avec le résultat du modèle de Rouse. Reproduit avec la permission de [11].

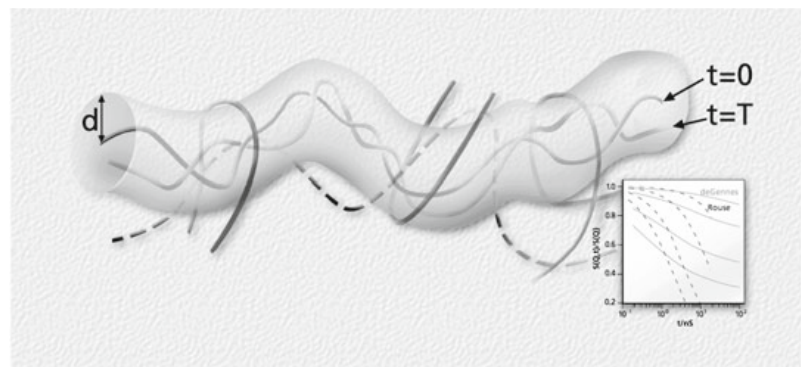

Figure 12. Vue d'artiste d'un tube virtuel constitué de chaînes de polymères entourant une autre.

de sa forte flexibilité qui permettent au modèle de Rouse d'être valide sur une large gamme de temps de $I(q, t)$.

Diverses tentatives ont été entreprises pour comprendre le changement des propriétés macroscopiques pour $M>M_{c}$. Le développement commence avec le modèle de tube d'Edwards [12] développé par de Gennes [13]. L'idée de base est que les molécules entourant le polymère forment un tube virtuel (figure 12) qui empêche le mouvement de la molécule de polymère centrale. Le seul mouvement qui est possible est celui le long de son propre contour, similaire au mouvement d'un serpent. C'est pour cette raison que le terme de "reptation"5 a été inventé pour ce type de mouvement.

Il doit être noté que le tube virtuel n'est pas collant mais restreint le mouvement d'une chaîne de polymère sur une échelle de taille intermédiaire $d \approx 5 \mathrm{~nm}$, plus grande que la dimension d'un monomère. C'est pourquoi aux temps courts cette restriction n'est plus active et le mouvement est de type Rouse. L'apparition de l'interaction du tube définit une nouvelle échelle de temps intermédiaire, le temps d'enchevêtrement $\tau_{e}$.

Dans ce modèle intuitif simple les résultats pour la viscosité et la diffusion peuvent être compris immédiatement. La viscosité d'un fondu de polymères est déterminée par le plus long temps de relaxation $\tau_{\eta}$, le temps nécessaire à un polymère pour quitter son tube initial et en constituer un nouveau. A l'intérieur du tube la chaîne suit une diffusion de Rouse avec $D_{\mathrm{R}} \propto N^{-1}$. La chaîne a complètement quitté sa configuration initiale lorsqu'elle s'est déplacée de sa longueur de contour $L=N \ell$.

\footnotetext{
${ }^{5}$ Du mot latin reptare $=$ ramper; la même racine que dans "reptile".
} 


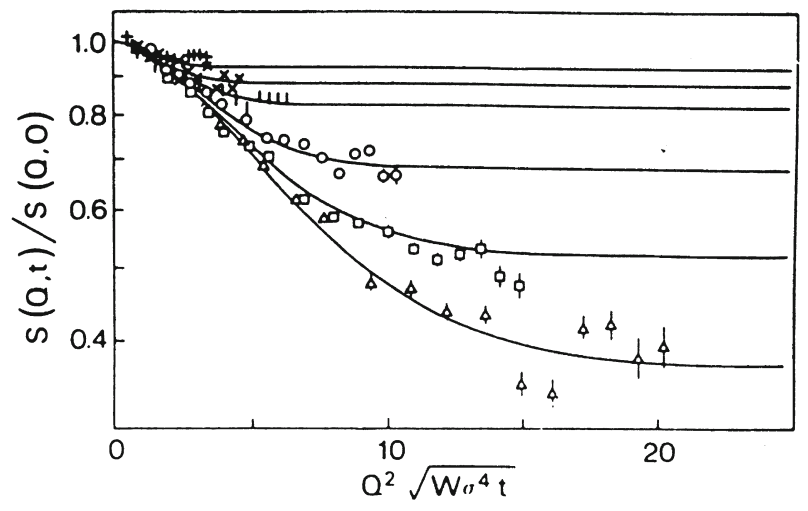

Figure 13. Spectres de spin-écho de polyethylenepropylene à $492 \mathrm{~K}$ tracés en représentation semilogarithmique et normalisés par la variable de Rouse comme sur figure 11. Les lignes continues sont des ajustements avec le modèle de Ronca. Reproduit avec la permission de [14].

(Notez qu'ici la restriction par le tube apparaît: Ce n'est pas la distance bout-à-bout $\sqrt{N} \ell$ qui compte mais la plus grande longueur de contour.) Donc on s'attend

$$
\eta \propto \tau_{\eta} \propto L^{2} / D_{\mathrm{R}} \propto N^{3}
$$

à une augmentation à la puissance trois de la viscosité avec le poids moléculaire. L'exposant réel est plus proche de 3.4 dans les expériences mais il y a des indications que pour les chaînes de très grande longueur la limite de 3 soit atteinte.

Dans l'espace réel, le contour de la chaîne suit une marche aléatoire Gaussienne, i.e. durant le temps $\tau_{\eta}$, il se déplace par diffusion sur une distance comparable à la distance bout-à-bout $R_{E} \propto \sqrt{N}$. Il est possible d'obtenir de ceci la constante d'auto-diffusion de la chaîne entière

$$
D \propto R_{E}^{2} / \tau_{\eta} \propto N^{-2}
$$

en parfait accord avec les expériences.

Bien que l'explication des résultats expérimentaux par le modèle de reptation soit assez satisfaisante, il est nécessaire de vérifier directement le comportement moléculaire pour une confirmation réelle du modèle. Un test sur des polymères réels est encore possible par la technique de spin-écho. La figure 13 montre les résultats sur du polyethylenepropylene (PEP) en utilisant le même mélange de polymères hydrogénés et deutérés qu'avant sur le figure 11. Il apparaît que la fonction intermédiaire de diffusion normalisée $I(q, t) / I(q, 0)$ ne peut être normalisée par la variable de Rouse (22). A la place d'une décroissance exponentielle aux temps longs, sa valeur tend vers un plateau.

Ceci peut être compris qualitativement comme un effet de restriction due au tube virtuel (figure 14). Pour les temps courts le mouvement de la chaîne obéit à la diffusion de Rouse. Sa position est en moyenne lisée et en conséquence la fonction de corrélation décroît. Dès que le déplacement moyen est de l'ordre du rayon du tube $\left(t=\tau_{e}\right)$ ce processus s'arrête et la fonction de corrélation ne se réduit plus. La valeur limite $I\left(q, t \gg \tau_{e}\right) / I(q, 0)$ décrit la forme du tube comme $S(q)$ pour un objet statique. Mais il est à noter que le tube "existe" seulement dans un certain régime de temps $\tau_{e} \ll t \ll \tau_{\eta}$ et ne peut donc être détecté par des méthodes de diffusion statique.

Pour une comparaison quantitative, il est nécessaire d'avoir des modèles analytiques qui puissent être comparés avec les résultats. Trois catégories différentes de modèles vont être discutées sans les expliquer en détail :

1. Dans les modèles appelés Rouse généralisés [15, 16] l'effet de gène topologique est décrite par une fonction mémoire. Dans le cas limite des longues chaînes les facteurs de structure dynamique 

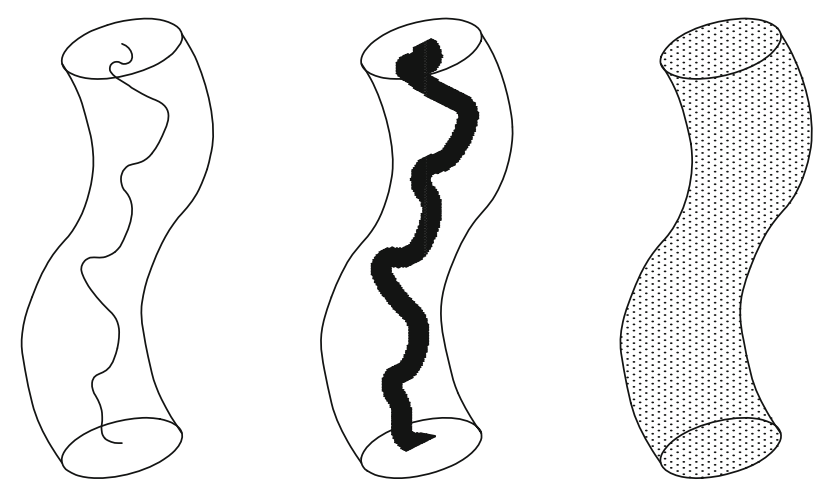

Figure 14. Représentation schématique de la dynamique d'une chaîne de polymère dans un tube formé de chaînes l'entourant, de gauche à droite: 1. conformation initiale de la chaîne, 2. distribution des conformations pour les temps courts $t<\tau_{e}, 3$. distribution des conformations pour les temps longs $t<\tau_{e}$.

peuvent être calculés explicitement avec des modèles de ce type dans des domaines de temps qui sont directement comparables aux expériences de spin-écho. Les lignes continues tracées sur la figure 13 correspondent à des ajustements des données avec le modèle généralisé de Rouse de Ronca [15].

2. En négligeant le mouvement initial de Rouse qui détermine la partie commune initiale des résultats de la figure 13, de Gennes a calculé explicitement dans son modèle de reptation local, le mouvement collectif des chaînes dans le tube de localisation [17] :

$$
\begin{aligned}
\frac{I\left(q, t \gg \tau_{e}\right)}{I(q, 0)}= & \left(1-\exp \left(-\left(\frac{q d}{6}\right)^{2}\right)\right) \exp \left(\frac{k_{\mathrm{B}} T \ell^{2} q^{4} t}{12}\right) \operatorname{erfc} \sqrt{\frac{k_{\mathrm{B}} T \ell^{2} q^{4} t}{12}} \\
& +\exp \left(-\left(\frac{q d}{6}\right)^{2}\right) \frac{8}{\pi^{2}} \sum_{p=1,3, \ldots} \frac{1}{p^{2}} \exp \left(-\frac{p^{2} t}{\tau_{\eta}}\right) .
\end{aligned}
$$

Ici le premier terme décrit la reptation locale alors que le second terme représente celle en dehors du tube. En dépit de sa structure compliquée, l'équation (25) donne un résultat simple pour le plateau

$$
\tau_{e} \ll t \ll \tau_{\eta}: \quad \frac{I(q, t)}{I(q, 0)}=\exp \left(-\frac{q^{2} d^{2}}{36}\right)
$$

avec $\tau_{e}=d^{4} \zeta / 3 \pi^{2} \ell^{2} k_{\mathrm{B}} T$.

3. Finalement, des Cloizeaux a formulé un modèle analogue à celui d'un caoutchouc (un polymère avec des liaisons de réticulation chimiques fixes aux temps intermédiaires [18]. Il fait l'hypothèse qu'une chaîne effectue un mouvement de Rouse entre des points d'enchevêtrement fixés.

Puisque ces trois types de modèles ont des concepts sous-jacents similaires, les résultats numériques ne sont pas très différents pour des temps courts dans le régime enchevêtré $\tau_{e} \ldots \tau_{\eta}$. C'est pourquoi, pendant longtemps, il n'a pas été possible de les discriminer et de décider de celui qui est le plus proche de la vérité expérimentale. C'est seulement récemment qu'une percée dans la technologie du spin-écho a pu résoudre cette question [19]. La figure 15 présente des résultats de NSE (IN15, ILL) jusqu'á 170 ns ajustés par les modèles mentionnés précédemment. Tous les ajustements ont un seul paramètre libre (la longueur d'enchevêtrement $d$ ) qui est listé dans le tableau 5, ainsi que le $\chi^{2}$ de l'ajustement. Il apparaît clairement que l'expression de la reptation totale (25) permet la meilleure description. Vient ensuite la "reptation locale", i.e. seulement le premier terme de (25). Dans ce cas, l'ajustement un peu plus mauvais est une indication que l'échelle du temps de reptation $\tau_{\eta}$ a déjá une influence dans la fenêtre de temps 


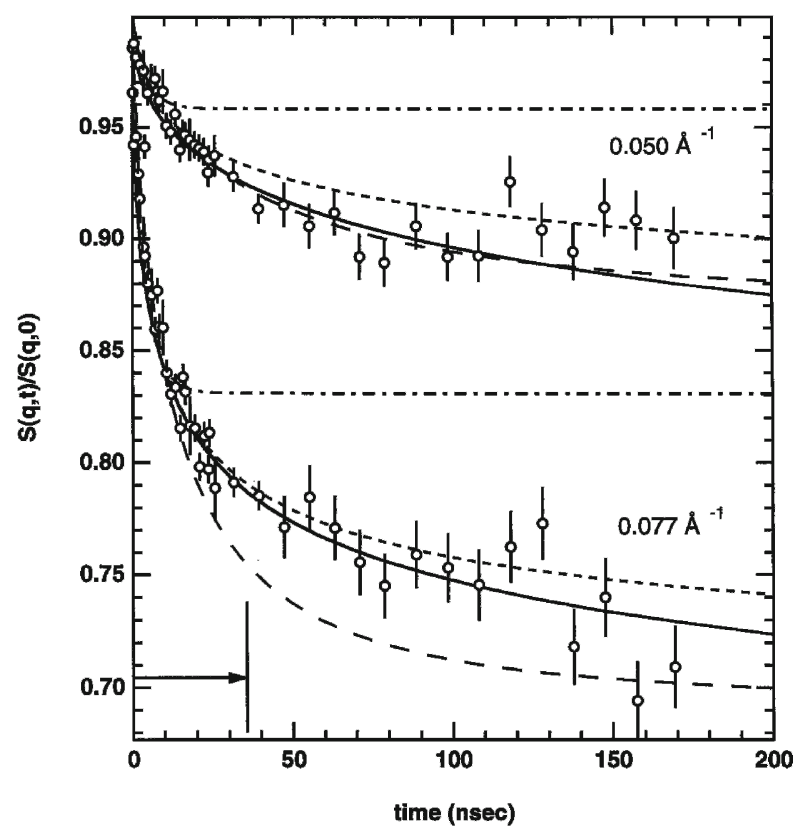

Figure 15. Spectres de spin-écho de polyethylenepropylene à $509 \mathrm{~K}$ pour des vecteurs d'onde $q=0.050$ et $0.077 \AA^{-1}$ avec une comparaison avec les modèles de reptation (ligne continue), reptation locale (ligne avec des petits pointillées), les modèles de des Cloizeaux [18] (ligne pointillée) et de Ronca [15] (ligne pointillée avec des points) [19]. La ligne verticale et la flèche indiquent la limite de temps supérieure des expériences précédentes. Reproduit avec la permission de [19].

Tableau 5. Résultats des ajustements pour la distance d'enchevêtrement $d$ pour les modèles étudiés [19]. Les $\chi^{2}$ réduits sont aussi indiqués.

\begin{tabular}{lccc}
\hline Model & Reference & $d[\AA]$ & Reduced $\chi^{2}$ \\
\hline Reptation & {$[17]$} & $46.0 \pm 0.1$ & 3.03 \\
Local Reptation & {$[17]$} & $46.5 \pm 0.1$ & 3.21 \\
des Cloizeaux & {$[18]$} & $59.8 \pm 0.2$ & 7.19 \\
Ronca & {$[15]$} & $47.4 \pm 0.1$ & 12.2 \\
\hline
\end{tabular}

d'observation. Les deux autres descriptions tombent significativement plus loin en ce qui concerne la dépendance en $t$ présentée ici ainsi que la dépendance en $q$ [19]. Il doit également être mentionné que le paramètre $d$ obtenu avec le modèle de reptation est le plus cohérent avec celui calculé à partir des mesures de rhéologie $d=42 \AA$ [20].

\subsection{Dynamique à échelle locale}

\subsubsection{Relaxation $\alpha$, transition vitreuse}

Il peut être noté que tous les calculs de la section 2.1 laissent une question ouverte: Quelle est la valeur (ou la dépendance en température) du coefficient de friction monomèrique $\zeta$ ? Toutes les équations donnant des résultats en variables macroscopiques telles que la viscosité ou le coefficient de diffusion contiennent $\zeta$ comme pré-facteur. Il n'est donc pas possible de calculer des valeurs absolues sans le connaître. 


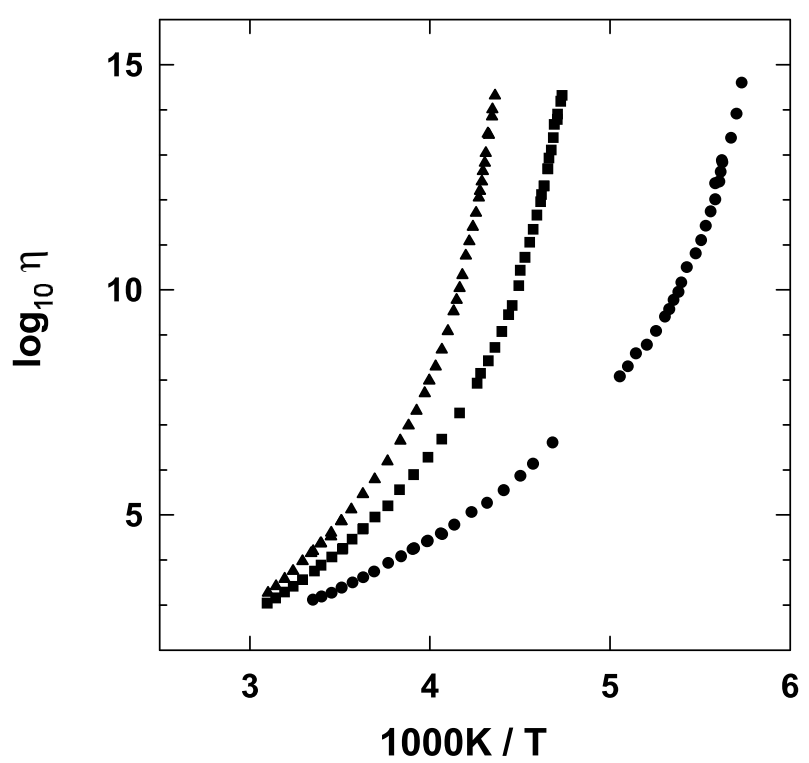

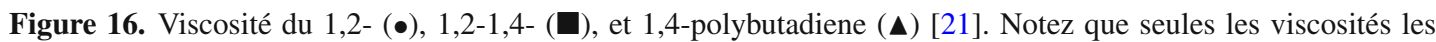
plus faibles $\left(<10^{5}\right)$ sont en fait mesurées sur un viscosimètre, les valeurs les plus élevées sont dérivées en utilisant le principe de superposition temps-température.

La figure 16 montre que c'est une question importante. La dépendance en température de la viscosité $\eta(T)$ est évidemment très forte et s'étend sur plusieurs décades sur une gamme de température comparativement faible. Les autres facteurs apparaissant dans $\eta(T)$ (e.g. $\ell, \rho, k_{\mathrm{B}} T$ ) montrent une dépendance en température beaucoup plus faible. La raison pour la forte dépendance en température de la viscosité (ou de la constante de diffusion, ou du temps de relaxation $\tau_{\eta}$ ) doit donc être $\zeta(T)$. Effectivement, $\zeta(T)$ présente une dépendance en température qui est très similaire à celle de la viscosité des liquides moléculaires de faibles masses qui ne cristallisent pas mais forment des verres. Comme le montre le tracé de type "activation" de la figure 16, il n'est pas possible de faire une description par une loi d'Arrhenius mais plutôt par des expressions phénoménologiques comme la loi de Vogel-Fulcher ${ }^{6}$

$$
\zeta(T) \propto \exp \left(\frac{B}{T-T_{0}}\right) .
$$

La similitude avec les liquides de faible poids moléculaire suggère que sur de faibles échelles spatiales, la dynamique des polymères n'est pas très différente de celle de ces derniers. Pour une observation directe de la dynamique des polymères, il est nécessaire d'effectuer des expériences de diffusion inélastique des neutrons à grand vecteur d'onde $q$ près du pic de corrélation moléculaire du facteur de structure, i.e. à environ $1.4 \AA^{-1}$. La figure 17 présente de tels résultats pour du polybutadiene [22], obtenus directement à partir de mesures de spin-écho. La figure 18 présente les mêmes résultats renormalisés par la viscosité et il apparaît que toutes les courbes $I(q, t) / I(q, 0)$ provenant des différentes températures coïncident. Ceci montre que la dynamique microscopique, à l' échelle d'un

${ }^{6}$ En science des polymères, la loi de Williams-Landel-Ferry ,équivalente mathématiquement, est souvent préférée

$$
\log _{10} \frac{\zeta(T)}{\zeta\left(T_{r}\right)}=\frac{-c_{1}^{0}\left(T-T_{r}\right)}{c_{2}^{0}+T-T_{r}}
$$

$T_{r}$ est une température arbitraire de référence. 


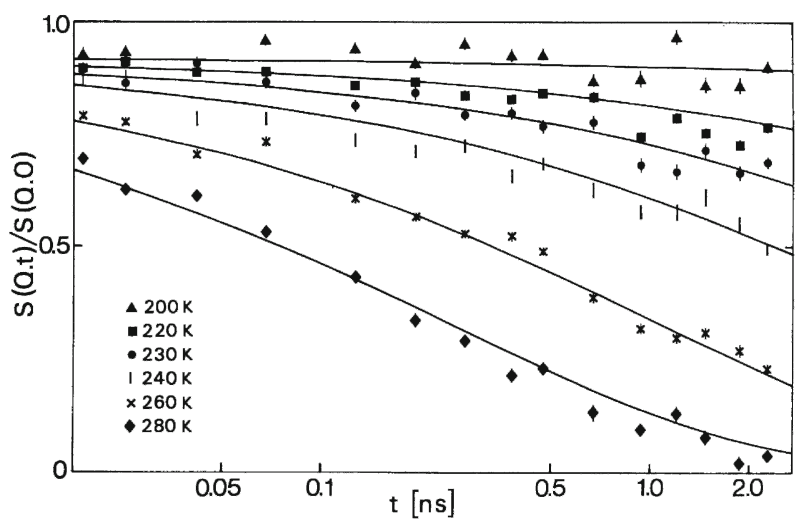

Figure 17. Spectres de spin-écho $\left(I_{\text {coh }}(q, t) / I(q, t)\right)$ de polybutadiene au même vecteur d'onde $q=1.48 \AA^{-1}$ qui corresponds au maximum du facteur de structure $S(q)$ [22]. Représentation logarithmique-logarithmique, l'axe des temps est normalisé par un temps $\tau$ dérivé des mesures de viscosité $(\tau \propto \eta)$. Les courbes sont des ajustements avec l'expression de Kohlrausch $\exp \left(-\left(t / \tau_{\mathrm{K}}\right)^{\beta}\right)$ avec $\beta=0.45$. Reproduit avec la permission de [22].

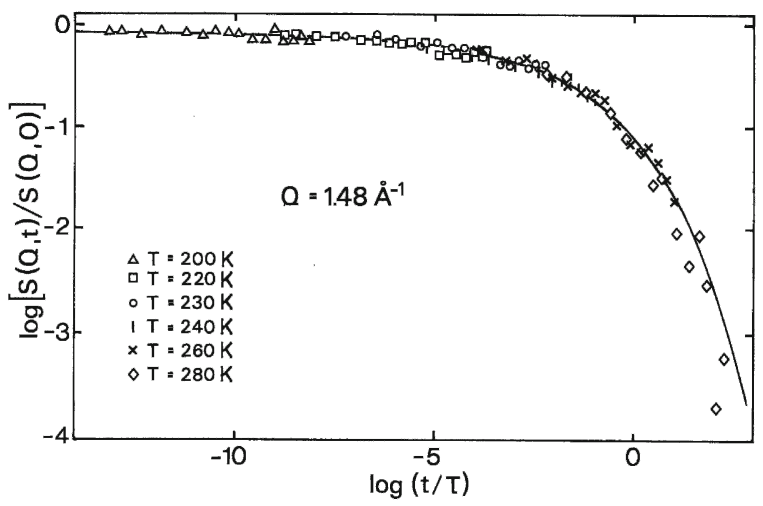

Figure 18. Mêmes résultats que sur la figure 17 mais en représentation logarithmique-logarithmique avec l'axe des temps normalisé par un temps $\tau$ dérivé des mesures de viscosité $(\tau \propto \eta)$. La courbe est un ajustement avec l'expression de Kohlrausch $\exp \left(-\left(t / \tau_{\mathrm{K}}\right)^{\beta}\right)$ avec $\beta=0.45$. Reproduit avec la permission de [22].

seul monomère, a en fait la même dépendance en température que la dynamique macroscopique au niveau d'une chaîne entière.

Néanmoins, des études postérieures à encore plus grandes valeurs de $q$ remettent en cause cette vision simple. Comme le montre la figure 19 , à $q=1.88 \AA^{-1}$ pour des températures inférieures à $220 \mathrm{~K}$, les courbes ne se placent pas sur la courbe maîtresse. La raison de cette déviation sera découverte plus tard et discutée dans la partie 2.2.3.

\subsubsection{Processus «rapides»}

Si on compare les résultats de spin-écho des figures 17 et 19 avec ceux de la partie 2.1, il est frappant que les courbes ne s'extrapolent pas à $I(q, t) / I(q, 0)=1$ aux temps courts. Cette contradiction apparente se résoud en considérent que nous ne voyons pas le régime des temps très courts (picoseconde) sur un spectromètre de spin-écho. Si un autre processus dynamique existe à de tels temps, il conduit à une décroissance de $I(q, t) / I(q, 0)$ pour des temps qui ne sont pas observables par spin-écho. 


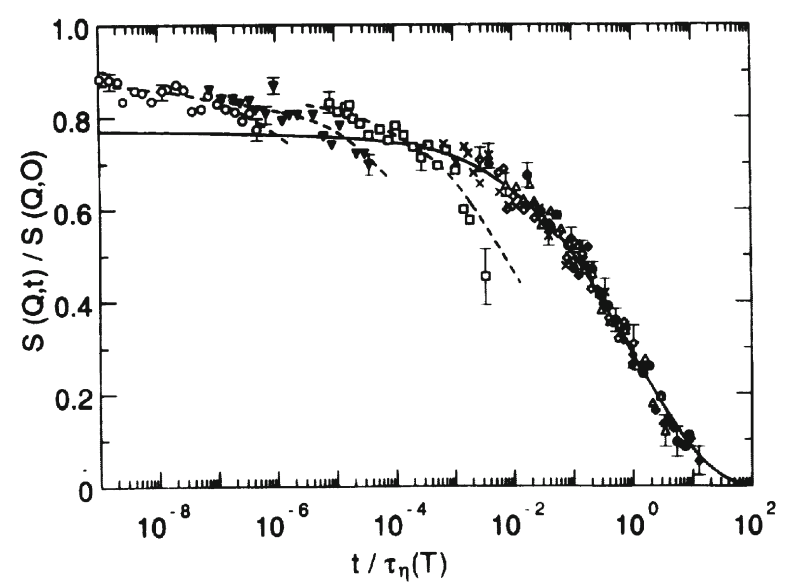

Figure 19. Spectres de spin-écho $\left(I_{\text {coh }}(q, t) / I(q, t)\right)$ de polybutadiene au vecteur d'onde $q=1.88 \AA^{-1}$ près du minimum du facteur de structure $S(q)$ [23]. L'axe des temps logarithmique est normalisé par un temps $\tau$ dérivé des mesures de viscosité $(\tau \propto \eta)$. Les courbes sont des ajustements avec l'expression de Kohlrausch $\exp \left(-\left(t / \tau_{\mathrm{K}}\right)^{\beta}\right)$ avec $\beta=0.37$.

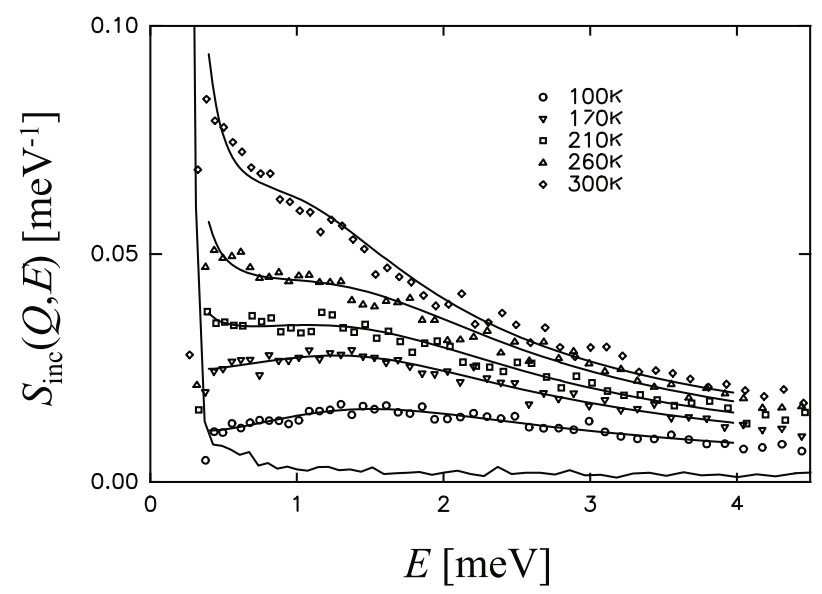

Figure 20. Spectres de TdV incohérents du 1,2-polybutadiene pour les températures indiquées et $q=2.2 \AA^{-1}$. La courbe épaisse est la fonction de résolution de l'instrument tracée à la même échelle. Les autres courbes sont des ajustements avec le modèle de vibration-relaxation [26].

En effet, les spectres de temps-de-vol (figure 20) montrent la présence de diffusion inélastique à des transferts d'énergie autour de $1 \mathrm{meV}$, ce qui corresponds à peu près à $2 \mathrm{ps}$, juste en dessous de la gamme de temps détectable par spin-écho.

Aux faibles températures, un pic dans $S_{\mathrm{inc}}(q, \omega)$ est visible qui est très similaire à celui observé dans les liquides de faibles poids moléculaire formant des verres (e.g. figure 4). En effet, ce pic appelé "Pic Boson" est caractéristique de systèmes formant des verres. Son nom est dérivé du fait que ce pic se normalise avec le facteur de Bose, ce qui indique que son origine provient de vibrations thermiquement excitées. Puisque pour la diffusion incohérente aux faibles températures $S_{\text {inc }}(q, \omega) \propto g(\omega) / \omega^{2}$, on devrait plutôt s'attendre à une ligne plate parce que la densité d'états dues aux ondes sonores est $g(\omega) \propto \omega^{2}$ (Debye). Mais dans les systèmes formant des verres, il y a toujours un excès de densité d'états [24], ce qui a récemment été mis en relation avec l'existence de modes mous localisés [25]. 


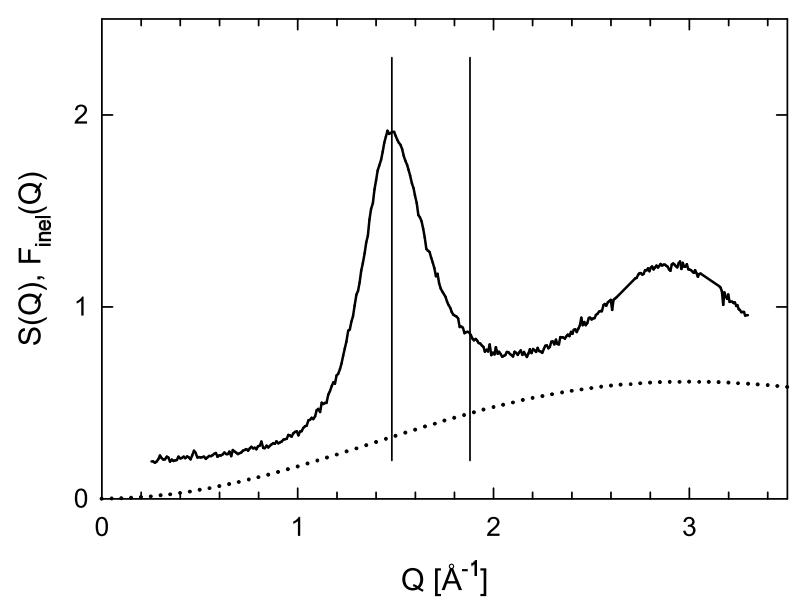

Figure 21. Facteur de structure $S(q)$ (ligne épaisse) et facteur de forme inélastique du modèle de sauts á deux sites avec $d=1.5 \AA$ (points). Les traits fins sont les vecteurs d'onde auxquels les expériences des figures 17 et 19 ont été réalisés, $q=1.48 \AA^{-1}$ et $1.88 \AA^{-1}$.

A des températures plus élevées, une intensité additionnelle apparaît, proche de la ligne élastique. La discussion de ce phénomène est encore controversée mais, comme le montrent les ajustements de la figure 20 , un modèle qui connecte les modes vibrationnels et le processus de relaxation par sauts permet de décrire les résultats avec succès [27,28].

\subsubsection{Relaxation secondaire $(\beta)$}

La déviation de $I(q, t) / I(q, 0)$ de la courbe maitresse visible sur la figure 19 a pu récemment être expliquée par l'existence d'une autre relaxation, la relaxation $\beta$. En utilisant la spectroscopie diélectrique, une telle relaxation "secondaire" a fréquemment été obtenue dans des systèmes variés formant des verres [29]. Elle est plus rapide que la relaxation $\alpha$ mais sa dépendance en température est plus faible, et peut généralement être décrite par une loi d'Arrhenius $\tau \propto \exp \left(E_{\mathrm{A}} / k_{\mathrm{B}} T\right)$. Ainsi, à une certaine température, les deux relaxations fusionnent.

Si on compare les résultats de spectroscopie diélectrique et de spin-écho, on trouve que les résultats de la figure 19 couvrent juste la région de recouvrement. C'est pourquoi ils doivent être décrits par un modèle prenant en compte les deux relaxations [30]. Sans présenter les détails des calculs nous donnons ici l'expression du résultat :

$$
\begin{aligned}
\frac{I(q, t)}{I(q, 0)}= & \exp \left(-\left(\frac{t}{\tau_{\mathrm{K}}(q, T)}\right)^{\beta}\right) \\
& \times\left(1-\frac{1}{S(q)} \int_{0}^{\infty} g\left(E_{\mathrm{A}}\right) F_{\text {inel }}\left(q, d\left(E_{\mathrm{A}}\right)\right) \mathrm{d} E_{\mathrm{A}}\right. \\
& \left.+\frac{1}{S(q)} \int_{0}^{\infty} g\left(E_{\mathrm{A}}\right) \exp \left(-\frac{2 t}{\tau\left(E_{\mathrm{A}}\right)}\right) F_{\text {inel }}\left(q, d\left(E_{\mathrm{A}}\right)\right) \mathrm{d} E_{\mathrm{A}}\right) .
\end{aligned}
$$

Le premier facteur décrit la relaxation $\alpha$ par une fonction de Kohlrausch $\exp \left(-\left(t / \tau_{\mathrm{K}}\right)^{\beta}\right)$. C'est la même forme de fonction que celle résultant du modèle de Rouse mais en général $\beta \neq 1 / 2 . \tau_{\mathrm{K}}(q, T)$ contient la dépendance en température exprimée par (27) et une dépendance en loi de puissance de $q$.

Le second facteur contient la relaxation $\beta$. Puisque il est supposé que les relaxations $\alpha$ and $\beta$ sont des processus indépendants, la fonction intermédiaire de diffusion résultante est un produit des 


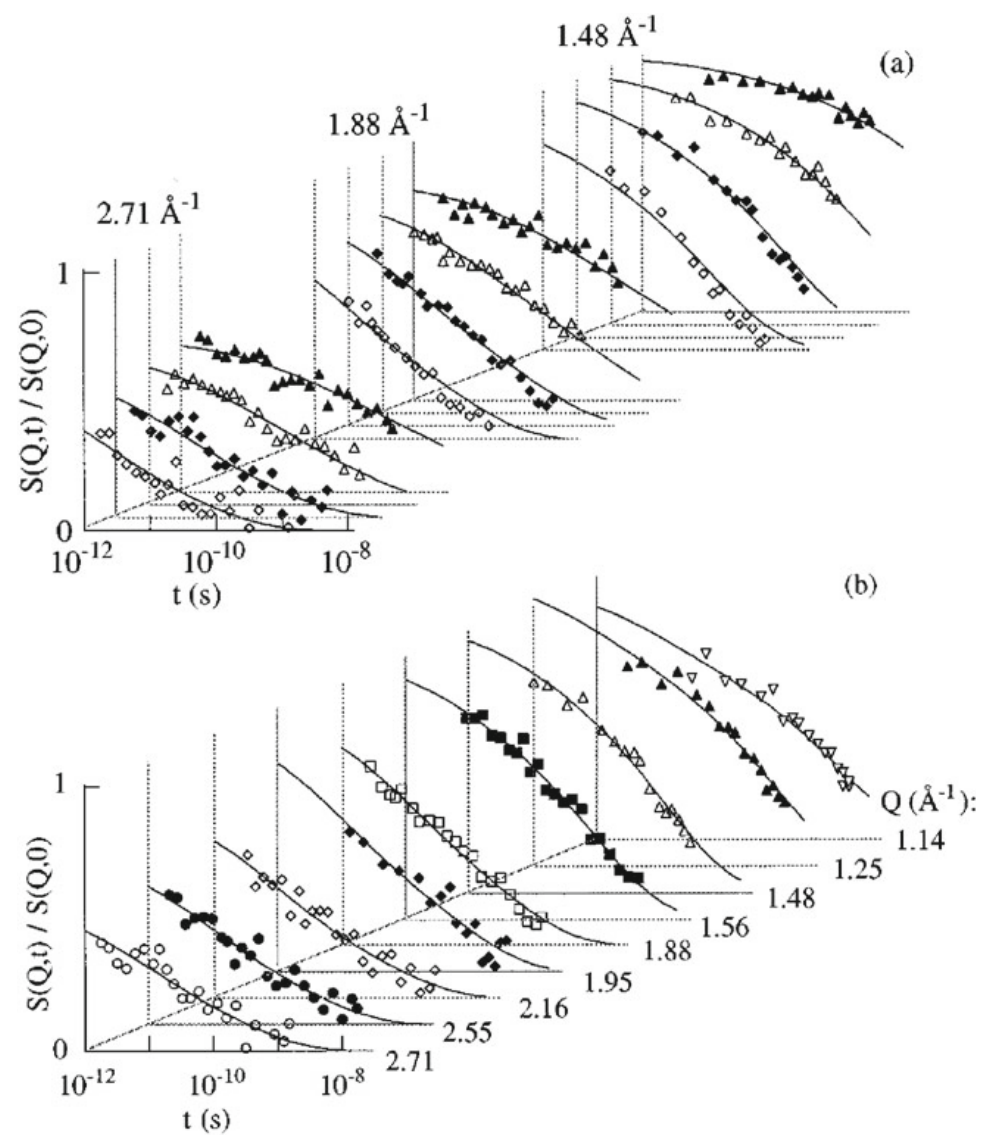

Figure 22. (a) Spectres de spin-écho du polybutadiene pour les valeurs de $q$ indiquées à $220 \mathrm{~K}(\boldsymbol{\Delta}), 240 \mathrm{~K}(\triangle)$, $260 \mathrm{~K}(\diamond)$, et $280 \mathrm{~K}(\diamond)$, (b) à $260 \mathrm{~K}$ pour les valeurs de $q$ indiquées. Les lignes continues sont des ajustements avec l'expression (28). Reproduit avec la permission de [30]

fonctions individuelles [3]. $S(q)$ est le facteur de structure d'une expérience de diffraction, pour la diffusion cohérente $I(q, 0)=S(q)$. $F_{\text {inel }}(q, d)$ est le facteur de forme inélastique du mouvement de la relaxation $\beta$. Dans le cas le plus simple, on peut faire l'hypothèse d'un saut entre les deux positions à la distance $d$,

$$
F_{\text {inel }}(q, d)=\frac{1}{2}\left(1-\frac{\sin q d}{q d}\right)
$$

mais des modèles moléculaires plus détaillés peuvent être conçus.

L'aspect temporel de la relaxation $\beta$ est inclus dans les intégrales sur les énergies d'activation. Comme la relaxation $\beta$ suit une loi d'Arrhenius, le temps de relaxation peut être exprimé comme $\tau\left(E_{\mathrm{A}}\right) \propto \exp \left(E_{\mathrm{A}} / k_{\mathrm{B}} T\right)$. Mais le fait qu'elle ne puisse être décrite par une exponentielle unique implique une distribution des énergies d'activation $E_{\mathrm{A}}$. Une telle description a été couronnée de succès pour les résultats de spectroscopie diélectrique [31] et la $g\left(E_{\mathrm{A}}\right)$ utilisée dans $(28)$ a été prise à partir des résultats de la fonction diélectrique. De plus la relation $d\left(E_{\mathrm{A}}\right) \propto E_{\mathrm{A}}^{1 / 4}$ a été utilisée, exprimant l'idée que les barrières d'énergie les plus élevées sont corrélées avec les distances les plus grandes du minimum de potentiel. 
De l'expression (28) il apparaît immédiatement qu'au maximum du $S(q)$ la relaxation $\beta$ est de moindre importance. La figure 21 montre repectivement les "poids" des relaxations $\alpha$ et $\beta, S(q)$ et $F_{\text {inel }}(q)$ (modélisé par un saut à deux sites avec $d=1.5 \AA$ ). Ceci explique les différences entre les figures 18 et 19 . Pour un vecteur d'onde de $1.48 \AA^{-1}$ la relaxation $\alpha$ est dominante alors qu'à $q=1.88 \AA^{-1}$ la relaxation $\beta$ est à peu près aussi importante. La figure 22 montre des expériences qui ont été réalisées pour beaucoup plus de vecteurs d'onde et de valeurs de température [30]. Elles peuvent toutes être modélisées de façon satisfaisante par le modèle (28).

\section{Remerciements}

L'auteur remercie Fabrice Cousin, Julian Oberdisse et Jacques Jestin pour la traduction de ce cours ainsi que Stéphane Longeville pour sa relecture attentive.

\section{Références}

[1] Lovesey, S. W. (1984): Theory of Neutron Scattering from Condensed Matter, Clarendon Press, Oxford.

[2] Squires, G. L. (1978): Introduction to the theory of thermal neutron scattering, Cambridge University Press, Cambridge.

[3] Bée, M. (1988): Quasielastic neutron scattering, Adam Hilger, Bristol.

[4] Mezei, F. (ed.) (1980): Neutron spin echo, Springer, Heidelberg.

[5] Ferry, J. D. (1980): Viscoelastic properties of polymers, John Wiley \& Sons, New York.

[6] Nemoto, N., Moriwaki, M., Odani, H. \& Kurata, M. (1971). Macromolecules 4, 215-219: "Shear Creep Studies of Narrow-DistributionPoly(cis-isoprene)".

[7] Berry, C. G. \& Fox, T. G. (1971). Adv. Polym. Sci. 5, 261-357: "The Viscosity of Polymers and their Concentrated Solutions".

[8] Pearson, D. S., Ver Strate, G., v. Merwall, E. \& Schilling, F. C. (1987). Macromolecules 20, 1133-1141: "Viscosity and Self-Diffusion Coeeficient of Linear Polyethylene".

[9] Rouse, P. E. (1953). J. Chem. Phys. 21, 1272-1280: "A Theory of the Linear Viscoelastic Properties of Dilute Solutions of Coiling Polymers".

[10] Doi, M. \& Edwards, S. F. (1986): The Theory of Polymer Dynamics, Clarendon Press, Oxford.

[11] Richter, D., Ewen, B., Farago, B. \& Wagner, T. (1989). Phys. Rev. Lett. 62, 2140-2143: "Microscopic Dynamics and Topological Constraints in Polymer Melts: A Neutron-Spin-Echo Study".

[12] Edwards, S. F. (1967). Proc. Phys. Soc. 92, 9-16: "The statistical mechanics of polymerized material".

[13] de Gennes, P. G. (1971). J. Chem. Phys. 55, 572-579: "Reptation of a Polymer Chain in the Presence of Fixed Obstacles".

[14] Richter, D., Farago, B., Fetters, L. J., Huang, J. S., Ewen, B. \& Lartigue, C. (1990). Phys. Rev. Lett. 64, 1389-1392: "Direct Microscopic Observation of the Entanglement Distance in a Polymer Melt".

[15] Ronca, P. F. (1983). J. Chem. Phys. 79, 1031-1044: "Frequency spectrum and dynamic correlations of concentrated polymer liquids".

[16] W. Hess (1988). Macromolecules 21, 2620-2632: “Generalized Rouse Theory for Entangled Polymeric Liquids".

[17] de Gennes, P. G. (1981). J. Phys. (France) 42, 735-740: “Coherent scattering by one reptating chain".

[18] des Cloizeaux, J. (1993). J. Phys. I (France) 3, 1523-1539: "Dynamic form function of a long polymer constrained by entanglements in a polymer melt".

[19] Schleger, P., Farago, B. \& Lartigue, C. (1998). Phys. Rev. Lett. 81, 124-127: "Clear Evidence of Reptation in Polyethylene from Neutron-Spin-Echo Spectroscopy". 
[20] Carella, J., Graessley, W. \& Fetters, L.(1984). Macromolecules 17, 2775-2786: "Effects of Chain Microstructure on the Viscoelastic Properties of Linear Polymer Melts: Polybutadienes and Hydrogenated Polybutadienes".

[21] Zorn, R., McKenna, G. B., Willner, L. \& Richter, D. (1995). Macromolecules 28, 8552-8562: "Rheological Investigation of Polybutadienes Having Different Microstructures over a large Temperature Range".

[22] Richter, D., Frick, B. \& Farago, B. (1988). Phys. Rev. Lett. 61, 2465-2468: "Neutron-Spin-Echo Investigation on the Dynamics of Polybutadiene near the Glass Transition".

[23] Richter, D., Zorn, R., Farago, B., Frick, B. \& Fetters L. J. (1992). Phys. Rev. Lett. 68, 71-74: "Decoupling of Time Scales of Motion in Polybutadiene Close to the Glass Transition".

[24] Inoue, K., Kanaya, T., Ikeda, S., Kaji, K., Shibata, K., Misawa, M. \& Kiyanagi, Y. (1991). J. Chem. Phys. 95, 5332-5340: "Low-energy excitations in amorphous polymers".

[25] Buchenau, U., Pecharroman, C., Zorn, R. \& Frick, B. (1996). Phys. Rev. Lett. 77, 659-663: "Neutron Scattering Evidence for Localized Soft Modes in Amorphous Polymers".

[26] Zorn, R. , Kanaya, T., Kawaguchi, T., Richter, D. \& Kaji, K. (1996). J. Chem. Phys. 105, 11891197: "Influence of the microstructure on the incoherent neutron scattering of glassforming polybutadienes".

[27] Buchenau, U., Schönfeld, C., Richter, D., Kanaya, T., Kaji, K. \& Wehrmann, R. (1994). Phys. Rev. Lett. 73, 2344-2347: "Neutron Scattering Study of the Vibration-Relaxation Crossover in Amorphous Polycarbonates".

[28] Zorn, R., Arbe, A., Colmenero, J., Frick, B., Richter, D. \& Buchenau, U. (1995). Phys. Rev. E 52, 781-795: "Neutron scattering study of the picosecond dynamics of polybutadiene and polyisoprene".

[29] Johari, G. P. \& Goldstein, M. (1970). J. Chem. Phys 53, 2372-2388: "Viscous Liquids and the Glass Transition. II. Secondary Relaxations in Glasses of Rigid Molecules".

[30] Arbe, A., Richter, D., Colmenero, J., Farago, B. (1996). Phys. Rev. E 54, 3853-3869: "Merging of the $\alpha$ and $\beta$ relaxations in polybutadiene: A neutron spin echo and dielectric study".

[31] Hofmann, A., Alegrí a, A., Colmenero, J., Willner, L., Buscaglia, E. \& Hadjichristidis, N. (1996). Macromolecules 29, 129-134: "Secondary and Segmental Relaxation in Polybutadienes of Varying Microstructure: Dielectric Relaxation Results". 